\title{
Operationalizing diagnostic criteria for Alzheimer's disease and other age-related cognitive impairment—Part 2
}

\author{
Sudha Seshadri ${ }^{\mathrm{a}, \mathrm{b}, *}$, Alexa Beiser ${ }^{\mathrm{a}, \mathrm{b}, \mathrm{c}}$, Rhoda Au $^{\mathrm{a}, \mathrm{b}}$, Philip A. Wolf ${ }^{\mathrm{a}, \mathrm{b}}$, Denis A. Evans ${ }^{\mathrm{d}, \mathrm{e}}$, \\ Robert S. Wilson ${ }^{\mathrm{f}, \mathrm{g}, \mathrm{h}}$, Ronald C. Petersen ${ }^{\mathrm{i}, \mathrm{j}, \mathrm{k}}$, David S. Knopman ${ }^{\mathrm{i}, \mathrm{j}}$, Walter A. Rocca ${ }^{\mathrm{i}, \mathrm{k}}$, \\ Claudia H. Kawas ${ }^{1, \mathrm{~m}, \mathrm{n}}$, Maria M. Corrada ${ }^{\mathrm{l}, \mathrm{m}}$, Brenda L. Plassman ${ }^{\mathrm{o}}$, Kenneth M. Langa ${ }^{\mathrm{p}, \mathrm{q}, \mathrm{r}}$, \\ Helena C. Chui ${ }^{\mathrm{s}}$ \\ ${ }^{a}$ Framingham Heart Study, Department of Neurology, Boston University School of Medicine, Boston, MA, USA \\ ${ }^{b}$ Framingham Heart Study, National Heart, Lung, and Blood Institute, Framingham, MA, USA \\ ${ }^{c}$ Department of Biostatistics, Boston University School of Public Health, Boston, MA, USA \\ ${ }^{d}$ Rush Institute on Healthy Aging, Rush University Medical Center, Chicago, IL, USA \\ ${ }^{e}$ Department of Internal Medicine, Rush University Medical Center, Chicago, IL, USA

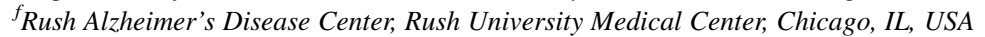 \\ ${ }^{g}$ Department of Neurological Sciences, Rush University Medical Center, Chicago, IL, USA \\ ${ }^{h}$ Department of Behavioral Sciences, Rush University Medical Center, Chicago, IL, USA \\ ${ }^{i}$ Department of Neurology, College of Medicine, Mayo Clinic, Rochester, MN, USA \\ ${ }^{j}$ Mayo Alzheimer's Disease Research Center, Mayo Clinic, Rochester, MN, USA \\ ${ }^{k}$ Division of Epidemiology, Department of Health Sciences Research, College of Medicine, Mayo Clinic, Rochester, MN, USA \\ ${ }^{l}$ Department of Neurology, University of California, Irvine, CA, USA \\ ${ }^{m}$ Institute for Memory Impairments and Neurological Disorders, University of California, Irvine, CA, USA \\ ${ }^{n}$ Department of Neurobiology and Behavior, University of California, Irvine, CA, USA \\ ${ }^{o}$ Department of Psychiatry and Behavioral Sciences, Duke University Medical Center, Durham, NC, USA \\ ${ }^{p}$ Division of General Medicine, Department of Medicine, School of Medicine, University of Michigan, Ann Arbor, MI, USA \\ ${ }^{q}$ Veterans Affairs Center for Practice Management and Outcomes Research, Ann Arbor, MI, USA \\ ${ }^{r}$ Institute for Social Research, University of Michigan, Ann Arbor, MI, USA \\ ${ }^{s}$ Department of Neurology, Keck School of Medicine, University of Southern California, Los Angeles, CA, USA
}

\begin{abstract}
This article focuses on the effects of operational differences in case ascertainment on estimates of prevalence and incidence of cognitive impairment and/or dementia of the Alzheimer type. Experience and insights are discussed by investigators from the Framingham Heart Study, the East Boston Senior Health Project, the Chicago Health and Aging Project, the Mayo Clinic Study of Aging, the Baltimore Longitudinal Study of Aging, and the Aging, Demographics, and Memory Study. There is a general consensus that the single most important factor determining prevalence estimates of Alzheimer's disease (AD) is the severity of cognitive impairment used as a threshold to define cases. Studies that require a level of cognitive impairment in which persons are unable to provide self-care will have much lower estimates than the studies aimed at identifying persons in the earliest stages of AD. There are limited autopsy data from the aforementioned epidemiological studies to address accuracy in the diagnosis of etiological subtype, namely the specification of $\mathrm{AD}$ alone or in combination with other types of pathology. However, other community-based cohort studies show that many persons with mild cognitive impairment and also some persons without dementia or mild cognitive impairment meet pathological criteria for $\mathrm{AD}$, thereby suggesting that the number of persons who would benefit from an effective secondary prevention intervention is probably higher than the published prevalence
\end{abstract}

All of the authors contributed equally to this article.

The authors have no conflicts to disclose. The sponsors had neither a role in the analysis or interpretation of these data, nor in the content of the paper. Appropriate approval procedures were used concerning human subjects.
*Corresponding author. Tel.: 617-414-1337; Fax: 617-638-8086.

E-mail address: suseshad@bu.edu 
estimates. Improved accuracy in the clinical diagnosis of AD is anticipated with the addition of molecular and structural biomarkers in the next generation of epidemiological studies.

(C) 2011 The Alzheimer's Association. All rights reserved.

Keywords: $\quad$ Alzheimer's disease; Dementia; Mild cognitive impairment; Cognitive impairment not dementia; Diagnostic criteria; Population-based; Prevalence; Incidence

\section{Introduction}

The U.S. national prevalence estimates of Alzheimer's disease (AD) have been produced using extrapolations from individual community-based studies and from combinations of them. They have also been obtained from a nationwide, cross-sectional study that relied on probability sampling to select individuals for inclusion. Regardless of the methodology, extrapolation, or probability sampling, the prevalence depends in large part on how cases are operationally defined. In a separate article in this journal issue, four sets of national prevalence estimates have been considered [1].

This article focuses on the diagnostic criteria used in the projects that were the basis for those prevalence estimates. Seshadri, Beiser, Au, and Wolf describe the diagnostic approach used in the Framingham Heart Study (FHS). Evans and Wilson describe the approach used in the East Boston Senior Health Project (EBSHP) and the Chicago Health and Aging Project (CHAP); both projects had similar case-finding strategies. Petersen, Knopman, and Rocca emphasize the threshold issue in diagnosis and provide an illustration using data from the Mayo Clinic Study of Aging (MCSA). Kawas and Corrada discuss their diagnostic experience with the Baltimore Longitudinal Study of Aging (BLSA) and provide a brief historic perspective on diagnosing dementia and AD in the Bronx Aging Study (BAS), the BLSA, and the 90+ Study. Plassman and Langa provide the operationalized diagnostic criteria for the Aging, Demographics, and Memory Study (ADAMS). Finally, in the Discussion section, Chui provide a synthesis of this material and some perspective.

\section{Prevalence of AD in the FHS: Influence of evolving diagnostic criteria}

The FHS, which began in 1948 as a prospective study of a community to identify risk factors for cardiovascular disease, has since grown into a three-generational study [2]. In 1976, a battery of neuropsychological (NP) tests was administered to the Original (generation [Gen] 1) cohort. This battery was a collection of tests designed by Edith Kaplan, Martin Albert, and Harold Goodglass to characterize baseline cognitive function in FHS participants [3]. The Folstein Mini-Mental State Examination (MMSE) has been administered since 1981 to allow for ongoing surveillance of cognitive status [4]. On the basis of these tests, a dementia-free cohort of 3349 subjects was established and has been followed up to the present. In 1971, offspring of subjects in the Original cohort and spouses of these offspring were enrolled into an Offspring (Gen 2) cohort, and in 1990, a diverse, multiethnic Omni cohort was added [5]. The dementia-free cohort for the Gen 2 was established in 1979 and had 4460 subjects. The Omni cohort was determined to be dementia-free at the time of study entry. These Gen 2 and Omni participants were added to the surveillance protocol for incident dementia.

Following referrals from FHS's core and ancillary studies and surveillance of performance on the MMSE, subjects suspected of cognitive impairment are evaluated by FHS physicians (neurologists and geriatricians), undergo NP testing, and are reviewed to determine whether the criteria for dementia are fulfilled. At this evaluation, dates of onset (earliest symptoms) and diagnosis (earliest date when diagnostic criteria are met), dates of transition in disease severity (mild to moderate to severe), dementia diagnosis, and subtype are designated. Over the years, cognitive status has been systematically tracked before and after the diagnosis of dementia, permitting fairly accurate ascertainment of the temporal evolution of cognitive impairment. As a corollary, greater precision is now possible in documenting the entire period of cognitive normality in each participant, thereby increasing person-years of follow-up and leading to more accurate lifetime risk estimates [6-11]. Recently, available data on all subjects were re-reviewed using information collected regularly from the following sources: FHS's core and ancillary examinations and health history updates; clinic, home, and nursing home assessments by the neurology and neuropsychology team; family interviews; records from medical contacts and nursing homes; and, when available, detailed brain autopsy findings gathered by the neuropathologist.

Initially only subjects with moderate or severe dementia had been included as dementia "cases," but this has now been expanded to include persons with mild dementia (all persons with a Clinical Dementia Rating score of 1 and some persons with a Clinical Dementia Rating score of 0.5) [12]. Lowering the functional threshold has increased the prevalence of dementia. For example, in the Gen 1 cohort, over the same study period between 1975 and 2008, 470 persons were recognized as developing incident dementia when case definition was restricted to persons who developed dementia of at least moderate severity, but this number increased to 504 persons when persons with mild dementia were included (Table 1). Thus, the age-specific prevalence of all-cause dementia at age 75 years almost doubled 
Table 1

Framingham Heart Study: Effect of varying severity of symptoms required to identify participant as having clinical dementia, and of varying intensity of tracking on the number of persons identified as "cases," and number censored for inadequate information

\begin{tabular}{|c|c|c|c|c|c|c|}
\hline $\begin{array}{l}\text { Period of } \\
\text { follow-up }\end{array}$ & $\begin{array}{l}\text { Outcome } \\
\text { criterion used }\end{array}$ & $\begin{array}{l}\text { Severity required to } \\
\text { make a diagnosis of } \\
\text { clinical dementia }\end{array}$ & $\begin{array}{l}\text { Total size of } \\
\text { cohort at risk }\end{array}$ & $\begin{array}{l}\text { Number of persons } \\
\text { identified as } \\
\text { dementia cases }\end{array}$ & $\begin{array}{l}\text { Number of persons } \\
\text { censored* before end } \\
\text { of study period }\end{array}$ & $\begin{array}{l}\text { Number of persons } \\
\text { who dies free of } \\
\text { dementia }\end{array}$ \\
\hline $1975-1985$ & Time to onset & Moderate + & 2391 & 102 & Data not obtained & Data not obtained \\
\hline \multirow[t]{2}{*}{ 1975-2008 } & Age at diagnosis & Moderate + & 2950 & 470 & 390 & 2090 \\
\hline & & Mild+ & 2930 & 504 & 467 & 1960 \\
\hline 1999-2004 & Cross-sectional & $\begin{array}{r}\text { Mild cognitive } \\
\text { impairment }\end{array}$ & 2778 & $630^{\dagger}$ & $-^{\dagger}$ & $--^{\dagger}$ \\
\hline
\end{tabular}

* Persons were censored if they developed dementia because of an alternate cause or their cognitive status was unknown at the end of the study period.

${ }^{\dagger}$ For these analyses, the presence or absence of mild cognitive impairment was defined using only objective performance criteria based on scores obtained at the first neuropsychological test administered to each participant between 1999 and 2004; persons with a score $\geq 1.5$ standard deviations below age-, gender-, and education-based norms were defined as having mild cognitive impairment.

between 1990 and 2005 (from 1.1\% to 1.9\%), and increased by approximately $25.0 \%$ even at 100 years of age $(44.4 \%-$ $57.4 \%$ ) using the revised threshold of mild dementia (Fig. 1). The 25-year and 40-year cumulative risks of incident dementia for 65-year-old persons, when estimated without reference to mortality because of competing causes, increased from $12.2 \%$ to $20.9 \%$, and from $62.3 \%$ to $66.6 \%$, respectively, when persons with mild dementia were included in estimates that had previously been based only on persons with moderate to severe dementia (Table 2). The corresponding residual lifetime risks adjusted for alternative cause mortality and hence reflecting the actual risk experience of the population also rose from $8.7 \%$ to $11.1 \%$ over a 25 -year follow-up period, and $13.5 \%$ to $15.3 \%$ over a 40-year period (Table 2).

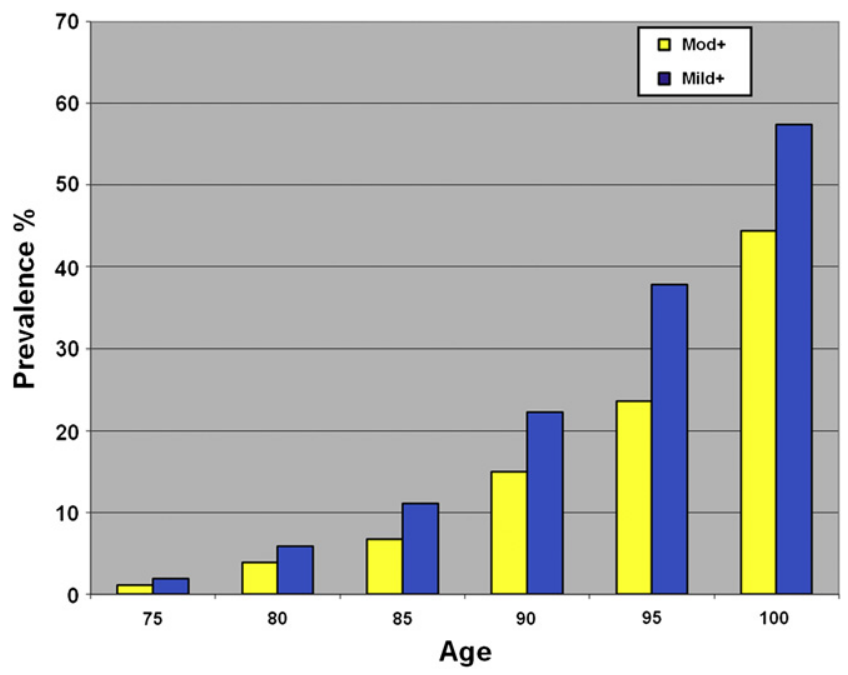

Fig. 1. Comparing varying estimates of the prevalence of dementia based on requiring different levels of severity (mild or moderate) to diagnose clinical dementia: Persons aged $\geq 65$ years from the Framingham Heart Study cohorts: 1990 to 2008. (Note: this is not a point or a period prevalence, but a prevalence estimated by assigning each cohort member a status as demented or not on the day he or she turned a given age [indicated on the $x$ axis.]). For interpretation of the references to color in this figure legend, the reader is referred to the Web version of this article.
With the evolution of diagnostic criteria for dementia and increasing interest in milder dementia and mild cognitive impairment (MCI), all cognitive and neurological data have been reassessed and current criteria applied. All individuals identified as having dementia satisfy Diagnostic and Statistical Manual of Mental Disorders (DSM)-IV criteria [13]. Persons categorized as $\mathrm{AD}$ are required to meet $\mathrm{Na}-$ tional Institute of Neurological and Communicative Disorders and Stroke (NINCDS)-Alzheimer's Disease and Related Disorders Association (ADRDA) criteria (NINCDS-ADRDA criteria) for possible, probable, or definite $\mathrm{AD}$ [14]. The diagnosis of vascular dementia $(\mathrm{VaD})$ is made on the basis of Alzheimer's Disease Diagnostic and Treatment Centers criteria [15] and National Institute of Neurological Disorders and Stroke (NINDS)-Association Internationale pour la Recherché et l'Enseignement en Neurosciences criteria [16], but the presence of $\mathrm{VaD}$ does not disqualify a participant from obtaining a concomitant diagnosis of AD if indicated. Diagnostic criteria for other types of dementia, such as Lewy body dementia and frontotemporal dementia, are also carefully specified on the basis of recently published criteria $[17,18]$.

\section{Table 2}

Framingham Heart Study: Comparing the 25-year and 40-year cumulative incidences (unadjusted for alternative cause mortality) and the 25-year and 40-year residual lifetime risks (adjusted for mortality due to competing causes) of Alzheimer's dementia in persons who are cognitively intact at age 65 years on the basis of variation in severity required to diagnose clinical dementia

\begin{tabular}{llll}
\hline Period of follow-up & $1975-1995$ & $1975-2008$ & \\
\hline $\begin{array}{l}\text { Outcome used } \\
\text { Severity required for } \\
\quad \text { clinical diagnosis }\end{array}$ & $\begin{array}{l}\text { Age at onset } \\
\text { Moderate }+\end{array}$ & $\begin{array}{l}\text { Age at diagnosis } \\
\text { Moderate }+\end{array}$ & $\begin{array}{l}\text { Age at diagnosis } \\
\text { Mild }+\end{array}$ \\
$\begin{array}{l}\text { Number demented } \\
\text { Cumulative incidence }\end{array}$ & 141 & 353 & 388 \\
$\quad$ & 14.4 & 12.2 & 20.9 \\
$\quad \begin{array}{l}\text { 25 years } \\
\quad 40 \text { years }\end{array}$ & 29.9 & 62.3 & 66.6 \\
$\begin{array}{l}\text { Residual lifetime risk } \\
\text { 25 years }\end{array}$ & 8.9 & 8.7 & 11.1 \\
$\quad 40$ years & 11.8 & 13.5 & 15.3 \\
\hline
\end{tabular}


In the past decade, MCI has emerged as an important concept defining a preclinical or prodromal stage of dementia, especially AD [19,20]. Detailed serial assessment of multiple neurocognitive domains and exploration for mild subjective symptoms can detect a pattern of asymptomatic or minimally symptomatic changes in performance on cognitive testing, which have been designated as pre-MCI and MCI, respectively. Defining MCI can help identify a population at high risk for developing dementia, especially AD. Relating risk factors and imaging and circulating biomarkers to $\mathrm{MCI}$ as well as mild AD can increase the ability to identify novel markers and to predict who progresses to overt clinical dementia. MCI has been divided into subtypes (amnestic and nonamnestic, with additional subcategories of single and multiple domain) that reflect the observed variability in early cognitive symptoms, including deficits in verbal memory and/or executive function, domains generally believed to reflect AD versus vascular pathology. Deficits in the cognitive domains of visuospatial memory, verbal learning, and verbal fluency have also been identified as indicators of impending clinical dementia [21].

Large epidemiological studies have typically defined MCI using objective performance criteria on the basis of quantitative NP test scores; cut-offs have been defined as $\geq 1.5$ standard deviations (SD) below age- and genderbased norms. FHS investigators have used their own community-based normative data to define possible MCI using objective psychometric criteria of domain-specific performance below age- and education-specific thresholds $(>1.5$ SD below the mean) for the sample (Table 1). On the basis of cognitive testing undertaken between 1999 and 2004 on all consenting and eligible FHS participants, and after exclusion of persons with clinical dementia, 630 individuals met criteria for possible MCI (a period prevalence of $22.7 \%$ ) with 192 individuals having single- or multipledomain amnestic MCI (period prevalence of 6.9\%).

Simultaneous NP examinations and magnetic resonance imaging scans enhance the ability to detect incidental or unsuspected neurological conditions such as brain tumors, subclinical vascular injury, or multiple sclerosis that could provide an alternative explanation for the observed NP test results. The imaging data also help to define the causes in persons diagnosed with MCI or dementia. However, the clinical diagnostic categories have now been extended to include MCI, and the FHS investigators identify this condition in two ways. In the initial articles, a purely objective psychometrically determined definition of MCI was used, sometimes called "possible" MCI and based on performance on an initial NP examination and classified into the four widely recognized subtypes $[19,20]$. However, since 2004 the FHS investigators began ascertaining clinically defined (or "probable") MCI by the incorporation of $\mathrm{MCI}$ in the dementia tracking and consensus diagnostic protocols, which include measures of functional as well as cognitive impairment.

These unique data may improve the ability to identify early MCI. Finally, since AD is being increasingly recog- nized as a life-course disease, FHS investigators are exploring the range of cognitive function among the young adults in the Gen 3 cohort using more sensitive tests than the MMSE (the Consortium to Establish a Registry for Alzheimer's Disease word list and a modified Stroop test) and will be able to assess if they can define cognitive profiles that may be called "Pre-MCI" [21] which track into middleand late-adult life.

\section{Implementation of criteria for the diagnosis of $A D$ in the population: Experience with the EBSHP and the CHAP}

How to implement criteria for the diagnosis of $\mathrm{AD}$ in the general population is not intuitively obvious but requires both conceptual and practical decisions on several key points. Estimates of AD prevalence from the EBSHP [22] and estimates of $\mathrm{AD}$ incidence from the CHAP [23] are higher than those reported from many but not all other studies. To a great extent, the differences result from these conceptual and practical decisions. The relevant issues and the choices made for the EBSHP and CHAP are briefly summarized in Table 3 and further discussed later in the text. In each instance, the rationale in the EBSHP and CHAP was to achieve greater accuracy and validity. However, each of the choices happened to favor higher rather than lower estimates of $\mathrm{AD}$ prevalence.

The spectrum of disease that a study considers clearly affects the prevalence estimates from the study, and some characteristics of $\mathrm{AD}$ create challenges. The clinical and pathological features of $\mathrm{AD}$, similar to those of most common chronic diseases of aging, typically arise by minute degrees over a period. Both clinically and pathologically, the division between normality and disease is continuous (illustrated in Fig. 2A). The characteristics of AD do not form a distribution that is distinct from that of normality (illustrated in Fig. 2B). However, the most generally used ways to describe the occurrence of $\mathrm{AD}$ are categorical and require that a diagnostic cut-point be placed in the continuum between normality and disease. Because it is not clear exactly where this cut-point should be placed, different investigators and the most skilled clinicians, each using the same diagnostic criteria with intelligence and good judgment, will place the cut-point differently. Further, mild disease that is difficult to separate from normality is common. As a result, minor differences in where the cut-point is placed can strongly affect the resulting estimates of $\mathrm{AD}$ incidence and prevalence, as illustrated by Figs. 2C, D.

Thus, variation in $\mathrm{AD}$ prevalence estimates is to some extent unavoidable, inherent to the challenge of attempting to summarize the frequency of a disease that forms a continuous distribution with normality using a simple yes/no classification scheme. Studies have varied in how they have confronted this challenge. Some studies, wanting to avoid any doubt that those diagnosed as having $\mathrm{AD}$ do indeed suffer from the disease, have considered only more advanced 
Table 3

Key considerations in implementing diagnostic criteria for Alzheimer's disease in population research: East Boston Senior Health Project and Chicago Health and Aging Project

\begin{tabular}{|c|c|c|}
\hline Issue & EBSHP and CHAP approach & $\begin{array}{l}\text { Effect of approach on } \mathrm{AD} \\
\text { prevalence estimates }\end{array}$ \\
\hline Spectrum of disease considered? & Wide: mild to severe & Higher \\
\hline Include disease risk factors or consequences in criteria? & No risk factors or consequences in criteria & Higher \\
\hline Selection for second-stage clinical evaluation? & Stratified random sampling from all strata of performance & Higher \\
\hline Masking of clinical evaluators to first-stage results? & Mask & Higher \\
\hline
\end{tabular}

Abbreviations: AD, Alzheimer's disease; CHAP, Chicago Health and Aging Project; EBSHP, East Boston Senior Health Project.

disease. A potential disadvantage of this approach is that false-negative errors are likely to be increased, thereby artificially lowering prevalence estimates. The EBSHP and CHAP attempted to consider the full spectrum of $\mathrm{AD}$ from mild to severe. A potential disadvantage of this approach is that some normal subjects will be misclassified as having $\mathrm{AD}$, thereby artificially elevating prevalence estimates. Such misclassification does not seem to have been a substantial problem in either study, however, because follow-up of subjects has shown that the majority of those thought to have AD experienced the progressive cognitive decline characteristic of the disease [24,25].

A second issue is the extent to which a study restricts its consideration to "pure disease" in estimating AD prevalence by excluding those subjects who have other coexisting potential explanations for dementia. Such exclusivity is a feature of the widely used NINCDS-ADRDA diagnostic criteria [14]. The clinical diagnosis of probable AD in this system requires the absence of other disorders that are considered to be contributing to the progressive deficits in memory and cognition. A similar criterion must be met for a diagnosis of dementia of the Alzheimer type in the
DSM-IV criteria [13]. However, exclusive criteria pose two problems. First, AD prevalence is underestimated because coexistence of common diseases is not only logically possible but also highly frequent in the oldest age groups in which AD occurs most frequently [26,27]. A second, perhaps greater, issue is that exclusionary criteria assume that two diseases cannot coexist, thereby making a diagnosis of another dementing condition to some degree protective against a diagnosis of $\mathrm{AD}$. For example, a diagnosis of $\mathrm{VaD}$ could prevent a subject from having a diagnosis of $\mathrm{AD}$. In a study of sufficient size, the risk factors for the other dementing illness may become artifactually protective against $\mathrm{AD}$ because they occur more frequently among those with the other dementing condition.

A third issue is that the risk factors or consequences are sometimes included in disease criteria. Although this may seem reasonable, it can lead to major artifacts because doing so constitutes a self-fulfilling prophecy. The associations of the included risk factors or consequences with the disease are determined by the disease definition. Everyone with the disease has to have that risk factor or consequence, and
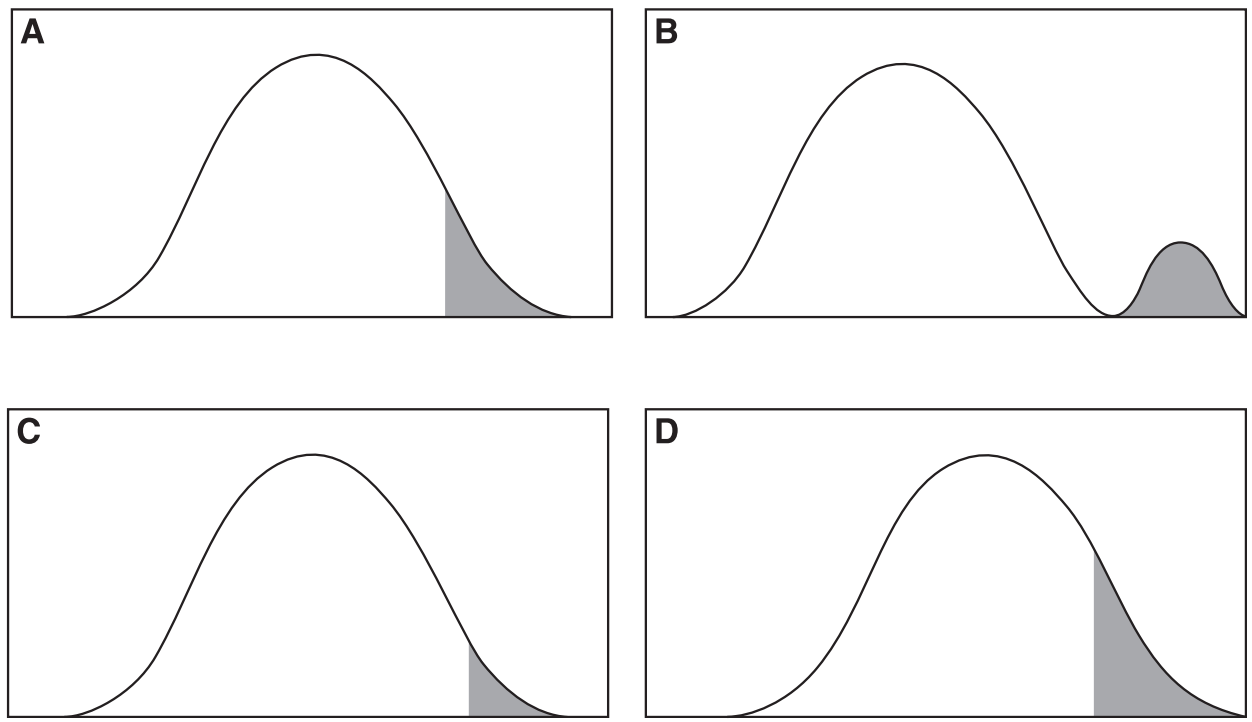

Fig. 2. Schematic representation of the distribution of Alzheimer's disease (AD) in a population. A is realistic, B is not. As shown in C and D, minor differences in where the cut-point is placed can strongly affect the resulting estimates of $\mathrm{AD}$ prevalence. 
an investigator cannot study the association of that risk factor or consequence with the disease. For example, impaired activities of daily living or altered patterns of behavior support a diagnosis of probable AD in the NINCDS-ADRDA system [14] and DSM-IV requires significant impairment in social or occupational functioning [13]. Therefore, it is not surprising that changes in activities of daily living and behavior will be associated with AD. To avoid these problems, the EBSHP and CHAP implementation of AD criteria have used the NINCDS-ADRDA criteria, but with two modifications. Diagnoses were not mutually exclusive, and no attempt was made to implement the supportive criterion regarding activities of daily living and patterns of behavior.

Several practical issues concerning study operations also strongly affect prevalence estimates. One is the method of selection of subjects for clinical evaluation by expert examiners. Many large-scale studies of AD are conducted in two stages. At the first stage, brief cognitive function tests are administered to all study subjects. Then, on the basis of the results of these tests, some subjects are selected for detailed clinical evaluation by skilled examiners. The motivations are typically cost saving (detailed clinical evaluation is expensive) and reducing the burden of study participation. In many studies, only subjects who fail the brief first-stage cognitive tests are evaluated at the second stage or, in addition, a very small proportion of those not failing the brief tests receive detailed evaluation. This approach assumes that all or almost all subjects who have AD will fail this first-stage screening. A major problem is that the utility of most brief cognitive tests as a screen for $\mathrm{AD}$ in the general population is typically unknown but very likely far from optimal. The effects are magnified as the fraction of subjects passing the first-stage brief test is usually high, typically about $85.0 \%$. Some studies avoid this problem, either by not using a first-stage test and administering detailed clinical evaluations to all subjects, or by randomly sampling from all levels of performance on the brief first-stage cognitive testing for detailed clinical evaluation. The EBSHP and CHAP adopted the latter approach, randomly sampling from all strata of first-stage performance for detailed clinical evaluation. If EBSHP sampling for detailed clinical evaluation had been confined to subjects in the worst performance stratum of first-stage brief cognitive testing, the resulting estimate of $\mathrm{AD}$ prevalence would have been one-half of the actual estimate [28], underscoring the magnitude of the issue.

A related point is that efficient masking or "blinding" of second-stage clinical evaluators to first-stage cognitive test results reduces the possibility of second-stage disease criteria being applied differently for those who did well and those who did badly at the first stage. Although the effects of masking of second-stage examiners to first-stage information on the resulting $\mathrm{AD}$ prevalence estimates are unknown, extensive experience with masking in clinical trials suggests that the effect may be substantial [29].

Another difficult operational issue is how certain an examiner has to be to make a diagnosis of AD. Typically, this issue takes the form of whether clinicians diagnosing $\mathrm{AD}$ must decide whether a subject meets criteria for $\mathrm{AD}$ or is normal, or whether they can use an intermediate category ("wiggle-room"). Typically, the forced-choice situation produces somewhat higher estimates of AD prevalence because more subjects having a high probability of $\mathrm{AD}$, but not complete certainty of the diagnosis, are assigned to the AD category. In the situation in which an intermediate category is available, some such subjects will be assigned to that category instead. The issue is often most apparent in situations in which a large consensus panel of knowledgeable clinicians is used, as it is likely that at least one or a few of them will have some reservation about a diagnosis of $\mathrm{AD}$ in such cases.

In conclusion, the positions taken on several conceptual and practical issues in a given study substantially affect the prevalence estimates produced by the study. In the EBSHP and CHAP, the positions on these issues were carefully considered and chosen before the studies were conducted. Each issue was resolved in the direction that best favored accurate and valid AD prevalence estimates. Although it was not a reason for choosing a position on any of these issues, each resolution also favored higher AD prevalence estimates (Table 3). Meaningful comparison of AD prevalence estimates across various studies would likely be enhanced by awareness of these and other issues that influence the estimates and by specification of how the issues were treated in each study.

\section{Diagnosing AD and dementia: Pre-MCI and now, thresholds are important (with illustration from the MCSA)}

The prevalence of $\mathrm{AD}$ varies among studies for a variety of reasons. A major reason is the thresholds used to define the clinical conditions. In earlier studies, there was a sharp demarcation set between normal cognition and dementia $[7,30]$. This was a reasonable approach and led to the boundary being set at a point on the cognitive continuum where a significant cognitive impairment was present to ensure the inclusion of only those subjects who were unquestionably impaired. As such, projects like the FHS counted moderate to severe cases as dementia, which then led to somewhat lower estimates of prevalence [7].

At the other extreme, investigators like those from the EBSHP estimated the overall prevalence of dementia to be $10.3 \%$, which was very age-related such that the estimate was reported to be $47.0 \%$ in the $\geq 85$ year group [22]. A salient feature of that study was the heavy reliance on objective cognitive testing to determine the presence of dementia. The other feature of dementia requiring an accompanying functional impairment was minimized in the EBSHP because of the difficulty in assessing that element of performance. The result of this decision was to place a heavy emphasis on cognitive testing and less emphasis on clinical judgment or the use of instruments to assess functional impairment. 
This decision led to a broader range of persons being counted as dementia cases.

The EBSHP prevalence figures were published in 1989, and, today, the issue of threshold used in that study could very well relate to the construct of MCI. MCI, particularly of the type that is considered to be the prodromal stages of $\mathrm{AD}$, constitutes a memory impairment beyond what one would expect for age in the setting of relatively preserved daily function [31]. Therefore, because the EBSHP investigators relied heavily on paragraph recall and discounted the role of functional impairment, their definition of dementia embraces the essence of what is being presently called as amnestic MCI. Thus, a difference between the FHS and the EBSHP was threshold of cognitive impairment set for dementia.

The issue of diagnostic thresholds relates to the constructs of sensitivity and specificity. As one moves the threshold back to lesser degrees of cognitive impairment, the increased sensitivity will capture larger numbers of persons; however, specificity will be compromised. That is, although many persons with the amnestic form of MCI of a degenerative origin are likely to be in the early stages of the AD process, not all will progress to AD [32]. Therefore, from a prevalence perspective, the numbers are larger, but they may be overestimates caused by over-inclusion of persons who may not have AD as the underlying pathophysiological substrate. Alternatively, as the threshold is moved toward a greater degree of cognitive impairment, the accuracy of the diagnosis with respect to correlating with underlying $\mathrm{AD}$ pathophysiology is higher, and consequently, specificity is high, but it is also likely that some early cases of true $\mathrm{AD}$ may have been missed (sensitivity is low).

Recent efforts are underway to address the problem of thresholds of cognitive impairment by linking the clinically milder cases such as those with amnestic MCI with imaging measures and biomarkers to enhance the specificity that a given clinical condition represents the underlying pathophysiology of $\mathrm{AD}$ [33-35]. One project directed toward this goal is the Alzheimer's Disease Neuroimaging Initiative (ADNI), which is a public-private partnership designed to recruit very mildly impaired subjects and predict who will progress to the dementia stage of $\mathrm{AD}$ through the use of neuroimaging and fluid biomarkers (in cerebrospinal fluid, plasma, or serum) [36,37]. This effort has been underway for approximately 5 years, and interesting data regarding the enhancement of clinical specificity through the use of imaging and other biomarkers have been reported [33]. The initial clinical focus of ADNI has been amnestic MCI and more recent iterations of this study have emphasized milder degrees of memory impairment. A similar effort in Europe, the Development of Screening Guidelines and Clinical Criteria for Predementia AD study, is evaluating the role of multiple predictors of progression in MCI [38].

The challenge for epidemiological studies lies in the implementation of these newer neuroimaging and biomarker techniques on a large scale. These measures tend to be quite expensive and may not be available in many epidemiological settings and, consequently, are often deemed not practical. However, as will be discussed later in the text, the MCSA is an attempt to address these issues through a populationbased random sample of nondemented subjects, using state-of-the-art neuroimaging and biomarker measures in a subset of subjects to determine how these measures perform in the general community setting [39]. Previous epidemiological studies performed at the Mayo Clinic using the medical records-linkage system of the Rochester Epidemiology Project likely had lower sensitivity at picking up the earlier stages of dementia [40].

Another issue that may influence the estimation of prevalence in the community is the underlying definition of $\mathrm{AD}$. Over the past several decades, AD has been defined as a clinical-pathological entity [41,42]. Although that was a reasonable definition in the $1980 \mathrm{~s}$, this approach is a source of confusion at present. More recent attempts to reformulate the constructs propose a clinical spectrum of cognitive impairment ranging from MCI through dementia and a corresponding pathophysiological substrate believed to be responsible for the clinical symptoms. However, the two spectra are kept separate. Therefore, when one grafts this approach onto an epidemiological study, one would discuss the prevalence of the clinical spectrum or the prevalence of the pathological features of the disorder, and they may not overlap completely [43-45]. Several studies have demonstrated the presence of the neuropathological substrate for $\mathrm{AD}$ in the absence of clinical symptoms [45]. Similarly, there are clinical studies of cognitive impairment consistent with a clinical diagnosis of $\mathrm{AD}$ in which the underlying neuropathology was variable [46-48]. More recent studies using molecular imaging techniques such as amyloid imaging will shed light on issues concerning the prevalence or at least the frequency of the amyloid substrate (abnormal amyloid deposition) in persons with and without clinical symptoms [49-51]. These studies will yield an index of the prevalence of the amyloid component of the pathological substrate of $\mathrm{AD}$, and will shed light on the value of this pathological feature in predicting the progression of $\mathrm{AD}$ pathology over time.

The MCSA is a population-based study of aging in Olmsted County, Minnesota. Initially, a random sample of 2000 persons aged 70 to 89 years was recruited [39]. Individuals known to be demented were enumerated and excluded from the prospective sample of nondemented subjects. The subjects each received a three-part evaluation as follows: clinical interview for medical history, family history, symptom onset, and a corresponding interview with an informant; a cognitive assessment consisting of nine tests in four cognitive domains yielding four domain scores and a composite $z$ score characterizing the combination of all four cognitive domains; and an examination by a physician for medical history, a mental status examination, and a neurological examination. The final clinical diagnosis was made by 

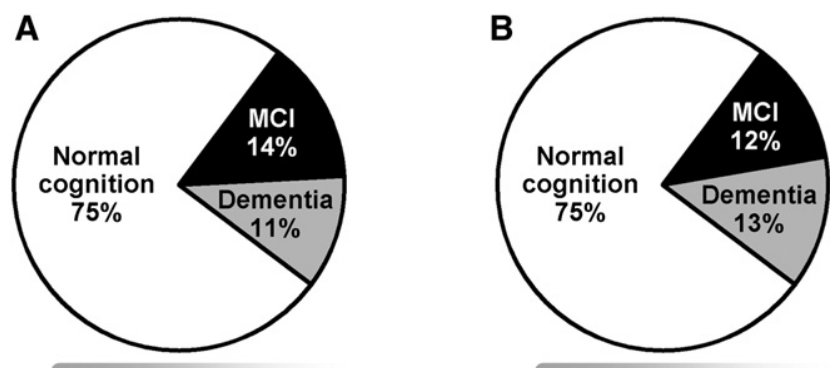

Dem $z=-2.4$
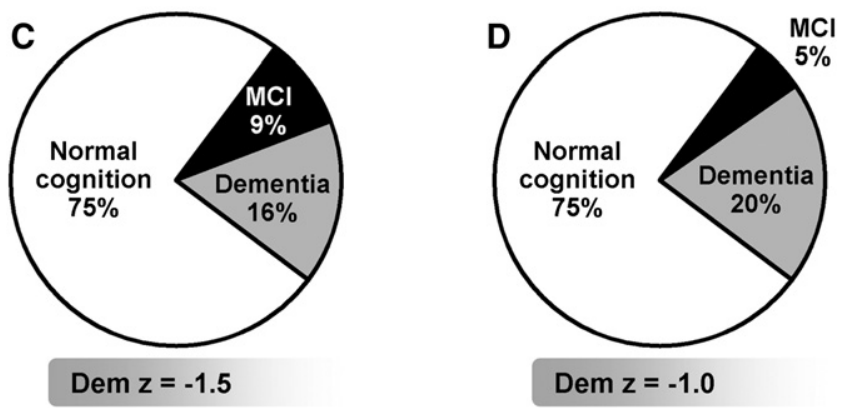

Estimates of $\mathrm{MCl}$ and dementia are age- and sex-adjusted to the Olmsted County, MN population

Fig. 3. The relative proportion of subjects classified as having dementia and mild cognitive impairment (MCI) in the Mayo Clinic Study of Aging. Panel A demonstrates the current division between $\mathrm{MCI}$ and dementia as determined by a consensus conference. The composite cognitive $z$-score is -2.4. Panels B, C, and D demonstrate the relative changes in proportions of cases with MCI and dementia as the degree of cognitive impairment moves toward the milder end of the spectrum.

a consensus of the three evaluators. On the basis of this assessment, the prevalence figures for cognitively normal, MCI, and dementia subjects were $75.0 \%, 14.0 \%$, and $11.0 \%$, respectively [52]. With this diagnostic categorization as a baseline, the global $z$-score representing the demarcation between MCI and dementia was -2.4. For demonstrating the effect of moving the threshold between MCI and dementia on the prevalence of both conditions, the global $z$-score was arbitrarily moved as shown in Fig. 3. When the $z$-score was moved from -2.4 to -2.0 , the prevalence of dementia increased to $13.0 \%$. As the threshold was moved further, the prevalence numbers changed accordingly. Therefore, this exercise demonstrates the importance of threshold on the underlying prevalence of dementia. As one moves toward the inclusion of subjects with lesser degrees of cognitive impairment in the dementia spectrum, the prevalence of MCI decreases, and the prevalence of dementia increases correspondingly.

In summary, the estimate of prevalence of a condition such as dementia or AD can be complex. Because the diagnosis is made on a clinical basis, the implementation of variable sets of criteria can influence the rates. Similarly, in addition to the specific instruments and measures used, the thresholds for defining the various conditions can vary.

As is shown in Fig. 4, the threshold for dementia has moved over the years. The earlier studies characterize AD

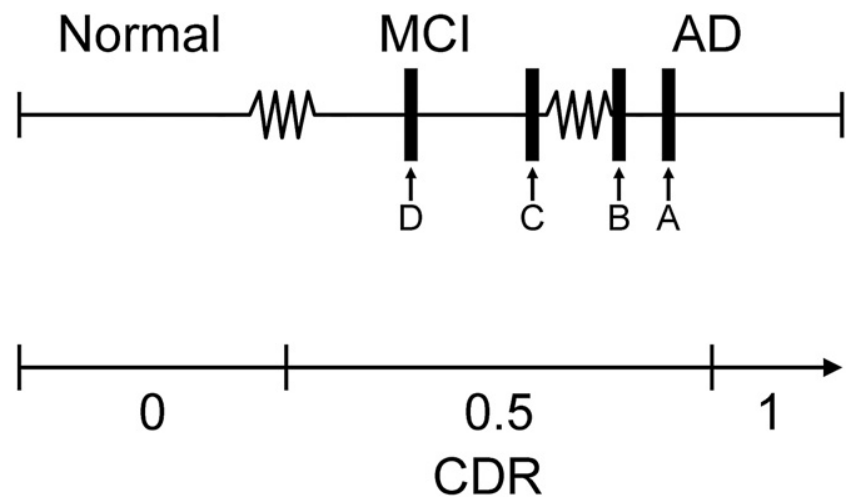

Fig. 4. A hypothetical threshold for differentiating mild cognitive impairment (MCI) from Alzheimer's disease (AD). Arrow A represents the older studies of prevalence of AD. Arrow B represents more recent studies but still characterizing completely developed dementia. Arrows $\mathrm{C}$ and D would result from the criteria for $\mathrm{AD}$ being moved into the current $\mathrm{MCI}$ range (see text).

in the clearly established range [7]. More recent studies, such as ADNI, suggest that, by adding imaging and fluid biomarkers to the clinical diagnosis of MCI, AD can be characterized at an earlier stage. As is shown in Fig. 4, the criteria for MCI in the original ADNI project, now called "late MCI," are marked by the arrow labeled "C." In the next phase of ADNI, the "early MCI" stage will be implemented, and it will result in a movement of the threshold to the arrow labeled "D" in Fig. 4. The EBSHP may have anticipated these changes. The value of imaging and other biomarkers may vary depending on the level of clinical certainty [53]. For example, biomarkers might become particularly important at the early MCI stage in making an etiological diagnosis of AD.

Finally, as the field moves toward an earlier identification of persons with dementia and $\mathrm{AD}$, the prevalence estimates are likely to increase. The construct of MCI, particularly amnestic MCI of a presumed degenerative origin, is very likely to represent the earliest clinical presentation of $\mathrm{AD}$, and consequently, if we move the threshold back to include these cases, while maintaining the specificity of the outcomes through biomarkers, the prevalence of dementia and $\mathrm{AD}$ will likely increase accordingly.

\section{The evolution of the diagnosis of dementia and AD: The BLSA experience and a brief historic perspective since the 1980s}

This section is divided into two distinct parts. The first part focuses on the BLSA, giving a brief description of the study, including the protocol for the dementia evaluations. In addition, the incidence results are mentioned and also characterized in terms of the special nature of the cohort. The second part is a brief historic perspective-with illustrations drawn from the BAS, the BLSA, and the 90+ Studyon how the diagnosis of dementia and AD has changed over the last 3 decades. Included are some observations on evaluating dementia in individuals of advanced age. 


\subsection{The BLSA experience}

The BLSA is a multidisciplinary, prospective study of normal aging conducted by the Intramural Division of the National Institute on Aging. The study began in 1958 and was initially limited to men, many of whom were retired government scientists. In 1978, enrollment of women began. The cohort consists of volunteers who tend to be welleducated. There are more men than women (two-thirds vs one-third), more whites than non-whites $(90.0 \%$ vs $10.0 \%$ ), and the age range is broad ( 21 to $>100$ years). Every 2 years, BLSA participants visit the center for 2.5 days of multidisciplinary investigations. The BLSA cohort does not represent the general aging population because of its ethnicity, high education, and volunteer nature.

Between 1985 and 1998, in addition to the usual BLSA protocol, a standardized neurological examination and NP testing were performed with the goal of determining prevalence and incidence of dementia and $\mathrm{AD}$, and to prospectively examine risk factors for AD. Active participants aged $\geq 65$ years received a neurological examination and NP battery, as described previously [30]. Participants aged 55 to 64 years were screened with the Blessed Information-Memory-Concentration (IMC) test [54], and those who made $\geq 3$ errors (out of a possible 33 ) also received the full neurological examination and NP evaluation. The cut-point of $\geq 3$ errors on the Blessed IMC test was conservatively chosen to maximize sensitivity at the expense of specificity. The procedural flow chart is shown in Fig. 5. Inactive BLSA participants were screened and examined with the same procedures, generally conducted in their homes throughout the United States. All screen-positive participants and $15 \%$ of screen-negative participants received complete evaluations. All procedures were administered biennially. In addition to neurological examinations and NP evaluations, case-detection methods included appropriate laboratory testing and neuroimaging, informant interviews, medical record review, and diagnostic consensus conference. DSM-III-Revised (DSM-III-R) criteria for dementia [55] and NINCDS-ADRDA criteria for AD [14] were applied.

During 9264 person-years of follow-up, 155 incident cases of dementia were identified, which were used to publish incidence rates [30]. Probable or possible AD accounted for $74.0 \%$ of the dementia cases, followed by vascular/mixed $\mathrm{AD}$ and $\mathrm{VaD}(10.0 \%)$, Parkinson's disease (7.0\%), and other dementias $(9.0 \%)$. Figure 6 shows age-specific incidence rates of $\mathrm{AD}$ in the BLSA and three other studies. The BLSA estimates fall in the middle of the range, higher than the FHS [6] and lower than the EBSHP [56]. However, the incidence estimates generated from the BLSA are likely to seriously underestimate the true incidence rate of $\mathrm{AD}$ in the study, and, even more so, the incidence in the general population, which is less educated than the BLSA participants and perhaps has a variety of health conditions that may increase the risk of dementia. Although poor health was not an explicit exclusion criterion for the BLSA, the study was conceived as an investigation of "normal aging." Moreover, participants were volunteers and had to be healthy enough to visit the center for evaluations. Thus, less healthy participants were more likely to refuse to participate or to drop out of the study. For the AD incidence and prevalence estimates, attempts were made to include participants who had become inactive by performing evaluations at their homes. However, under-representation of participants with poor health is likely to result in underestimates of incidence in the BLSA as well as other similar studies.

During the interval when 155 cases of dementia were identified, an additional 125 subjects who were classified as cognitive impairment not dementia (CIND) were found. As mentioned earlier, the dementia study was superimposed on the BLSA beginning in 1985 and continuing until 1998, with the methods described in this study. To maintain study integrity, procedures were put forth to limit diagnostic drift and other cohort effects. The NINCDS-ADRDA criteria had just been published and their application was still new to researchers. As compared with current practices, a higher level of cognitive impairment was required to make confident diagnosis of dementia and $\mathrm{AD}$ in 1985.

Incidence rates of dementia and AD in the BLSA are likely to be underestimates of the true $\mathrm{AD}$ incidence rates in the U.S. population. The conservative BLSA estimates reflect a highly educated group of volunteers who were primarily diagnosed with standards developed in the 1980s when dementia diagnoses were assigned at more severe levels of impairment. With the rapid aging of the worldwide population, numbers of individuals with dementia and $\mathrm{AD}$ will rapidly increase and present a growing public health problem.

\subsection{A brief historic perspective on evaluating for dementia and $A D$ since the 1980 s}

In 1983, Robert Katzman initiated one of the first prospective cohort studies of dementia, the BAS, funded by the National Institute on Aging and the NINCDS. Over the next 27 years, significant changes occurred in the diagnosis of dementia and AD. Dementia is now recognized at considerably milder clinical states, particularly in highly educated individuals, and the clinical-pathological accuracy has greatly improved.

In the early 1980s, normal participants were enrolled in the BAS if they had less than or equal to eight errors on the Blessed IMC test. Katzman selected the cut-off to be conservative in identifying a normal population in whom he could observe the development of dementia. Previous landmark studies by Blessed et al had identified 12 errors as a threshold for sufficient cognitive loss to be associated with amyloid/AD-type pathology [54]. However, the initial report from the BAS demonstrated that individuals with five to eight errors on the Blessed IMC test were highly likely to develop dementia within the next 3 years, compared with those with fewer errors. It was one of the first 


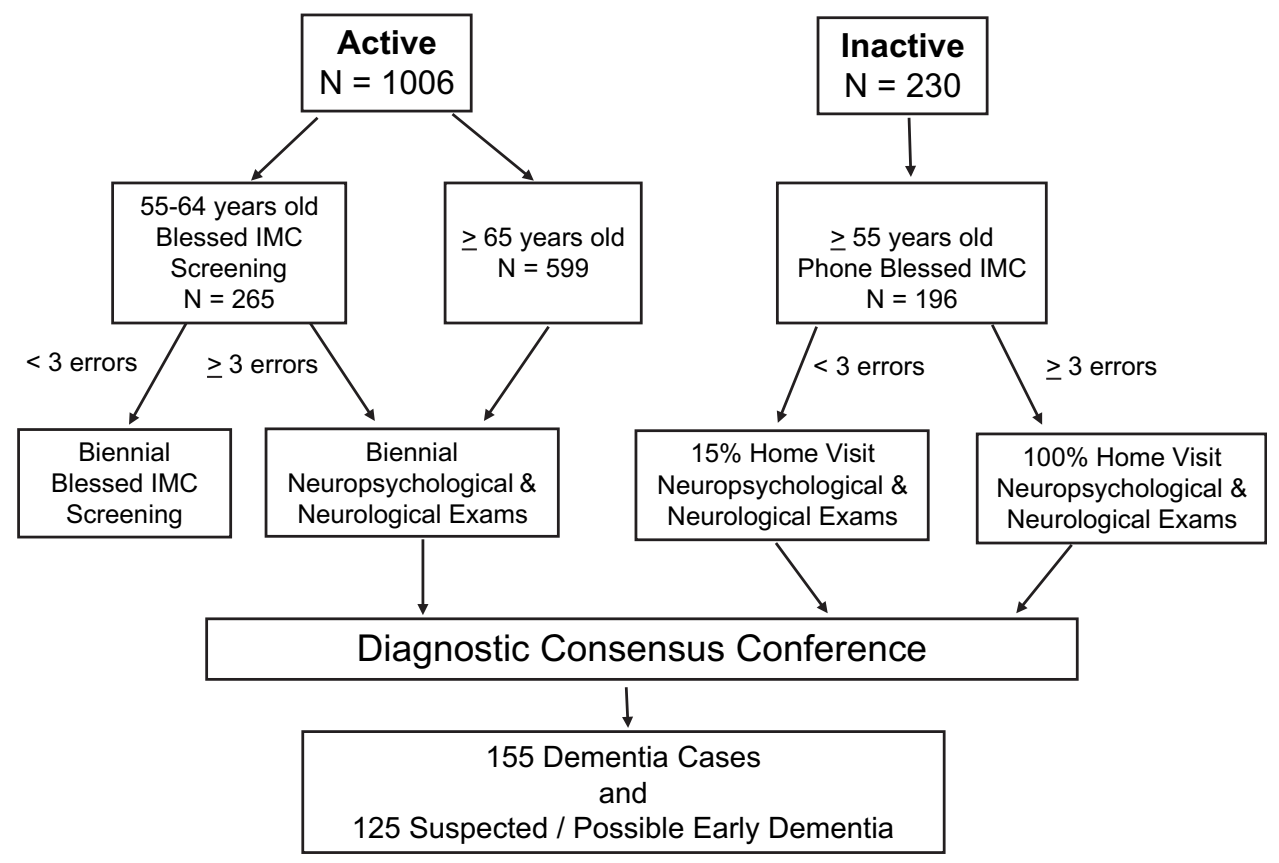

Fig. 5. Participant flow chart for the diagnosis of dementia in the Baltimore Longitudinal Study of Aging between 1985 and 1998 [30]. Abbreviations: IMC, Information Memory Concentration.

demonstrations that individuals with memory and other cognitive losses were likely in the "preclinical" stages of AD, akin to what is now called MCI. Katzman et al noted the importance of this finding because it allowed researchers to identify high-risk individuals who could then be targeted for therapeutic and other studies [57]. Many individuals with five to eight errors on enrollment, who were considered normal at that time, would no longer be considered "normal elderly," but rather to have MCI or even early AD.

Initially an attempt was made to assign $\mathrm{AD}$ diagnoses in demented subjects using recently proposed research diagnostic criteria [58]. Of the first 20 participants to develop dementia likely because of $\mathrm{AD}$, only two participants met the stringent research diagnostic criteria. Subsequently,

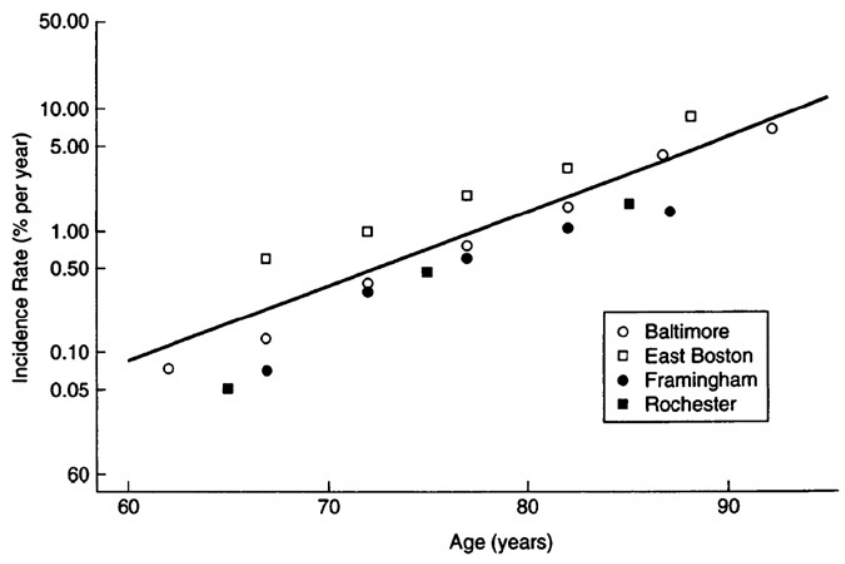

Fig. 6. Age-specific incidence rates of Alzheimer's disease on a log scale from four U.S. studies: Framingham, MA; East Boston, MA; Rochester, $\mathrm{MN}$; and Baltimore, MD. Reproduced with permission from Ref [43].
Katzman provided a draft of the NINCDS-ADRDA criteria, as yet unpublished. With these criteria, 20 cases of "probable or possible AD" out of approximately 30 incident cases of dementia were assigned. Most of the remaining cases were believed to have been caused primarily by vascular disease.

Given the experience of the BAS and the highly educated nature of the BLSA, a cut-off of three errors of the Blessed IMC was chosen to screen BLSA participants to receive complete evaluations. Because individuals developing dementia are more likely to become inactive, screening and evaluation of all inactive individuals were also done. It was frequently difficult to document cognitive and functional losses of sufficient severity to warrant a diagnosis of dementia in the highly educated BLSA participants, who in many cases had been going to the center for years. Nonetheless, many of these subjects were believed to be in early stages of dementia and thus were dubbed "suspects" or suspected dementia. This group, labeled CIND in Fig. 7, showed performance that was generally midway between normal and demented subjects. In some cases, it was a very poor performance, including a MMSE score below 20 and delayed recall scores in the MCI range. Furthermore, CIND subjects had problems with more cognitive domains than memory, as seen by the Trails B (executive function) and Animal Fluency (language) scores that were more similar to demented than to normal subjects (Fig. 7). However, incidence rates of dementia for these CIND participants were never estimated.

If some or all of the 125 suspected cases were added to the 155 incident cases, it would greatly increase the BLSA incidence estimates. Moreover, additional adjustment upward 
MMSE

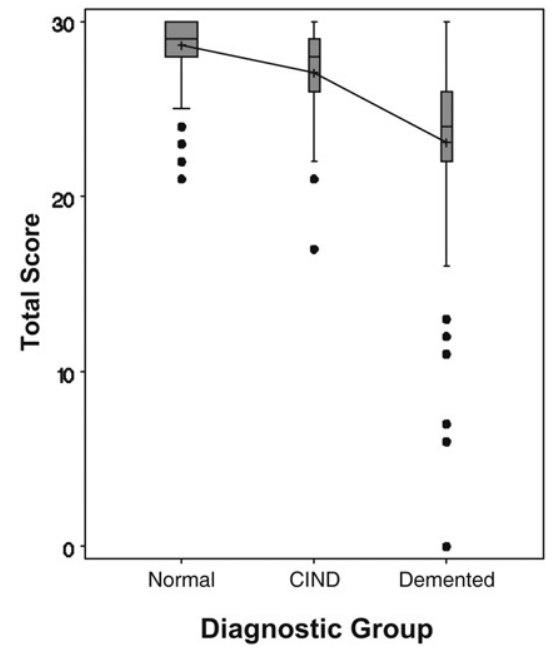

Trails B

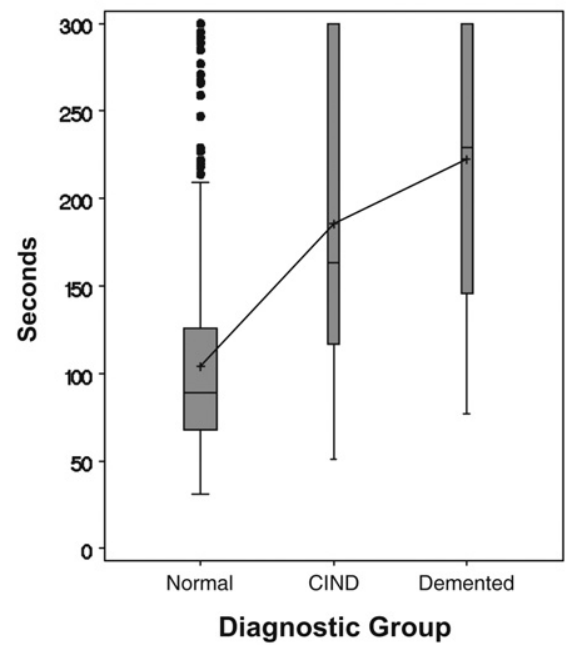

CSR Delayed Recall

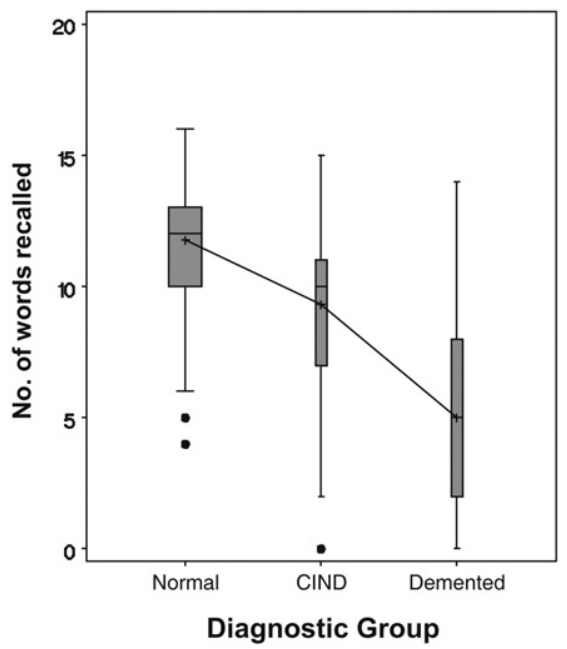

Animal Fluency

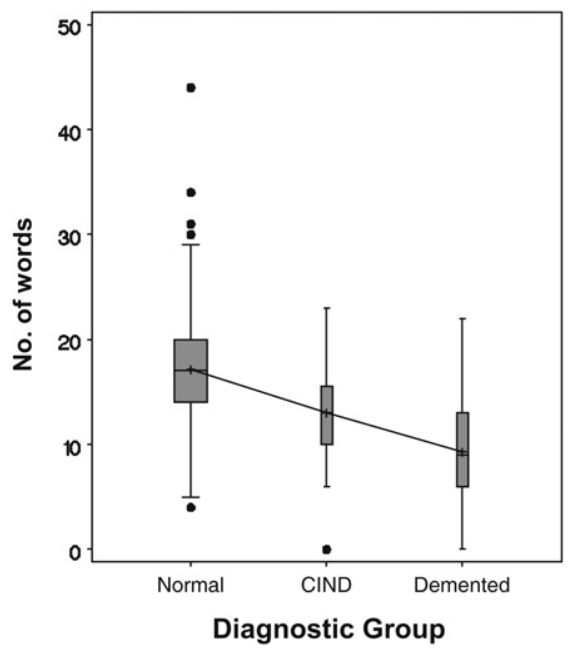

Fig. 7. Neuropsychological scores for Baltimore Longitudinal Study of Aging participants by clinical diagnosis group. Box width varies with sample size. Abbreviations: CSR, Cued Selective Reminding; CIND, cognitive impairment not dementia; MMSE, Mini-Mental State Examination.

would be necessary to generalize beyond this high-education and high-occupation group to the general population.

Initiated in 2003, the 90+ Study has enrolled more than 1500 people aged $\geq 90$ years in a prospective study of dementia and aging in the "oldest old." Participants were originally part of the 1981 Leisure World Cohort Study and resided in the same community. The sampling frame for the 90+ Study included all individuals from the Leisure World Cohort Study who were alive and aged $\geq 90$ years on January 1, 2003. To determine cognitive status, participants in the $90+$ Study received in-person evaluations, including a neurological examination, NP battery, medical history, and informant questionnaires, with evaluations taking place every 6 months at the participant's place of residence or in the research clinic. The age-specific prevalence [59] and incidence of dementia [60] in the oldest old have been estimated from this study. The incidence of dementia was found to continue to increase with age after 90 years of age, doubling every 5.5 years and reaching approximately $40.0 \%$ per year after 100 years of age. There was no difference in dementia incidence for women and men, but women live longer in general and probably live longer with dementia, thus, explaining their higher prevalence estimates $[59,60]$.

The diagnosis of dementia in people aged $\geq 90$ years has been challenging. High rates of sensory losses, fatigue, and medical comorbidities make it difficult to assess cognition, and normative values on NP tests have not been welldefined for this age group. Moreover, documentation of functional losses because of cognitive difficulties can be problematic in extremely aged individuals who generally are not working or taking complete responsibility for their living environment. To manage these challenges, it has been necessary to modify instruments in various ways, 
including larger stimuli, multimodal presentations, and additional questions during informant interviews.

The $90+$ participants have a lower education level and a mean age that is more than 20 years older than the BLSA participants. Thus, it would be expected that $90+$ participants would have lower normative scores. However, cognitive scores of normal participants in the $90+$ Study are similar to cognitive scores of normal BLSA participants, suggesting that cognitive impairment is identified at milder levels of decline in $90+$ subjects than was required for the younger BLSA participants between 1985 and 1998.

In the 90+ Study [61], as in other studies that examine the oldest old [62,63], dementia correlated poorly with traditional neuropathological markers of $\mathrm{AD}$, even in those with severe dementia. It is hoped that these observations will lead to improvements in the pathological and clinical diagnoses of dementia and $\mathrm{AD}$ at all ages (see also Mayeux et al [64] for further discussion of the diagnostic challenges in epidemiological studies of dementia and $\mathrm{AD}$ in the oldest old).

\section{Operationalizing diagnostic criteria in the ADAMS}

The ADAMS was designed to provide nationally representative data on the antecedents, prevalence, outcomes, and costs of dementia and CIND, using a unique studydesign based on the nationally representative Health and Retirement Study. The successful outcome of these aims is dependent on the assessment and diagnostic methods used to determine the diagnoses of dementia and CIND and their subtypes. Different assessment and diagnostic procedures may lead to different diagnostic outcomes for individuals participating in population-based studies of dementia. In this section, the operationalized diagnostic criteria for $\mathrm{AD}$, dementia, and CIND used in the ADAMS are described and information on the validation of the diagnostic procedures is provided.

The ADAMS, which included a sample of 856 individuals aged $\geq 71$ years from all regions of the country, used a single standardized assessment and diagnostic protocol that was modeled on the approach developed by a group of researchers common to ADAMS and three other epidemiological studies of cognitive aging [65-67]. The clinical assessment was designed to simulate a clinical evaluation for cognitive problems, with the exception that laboratory tests and neuroimaging were not performed as a part of the ADAMS protocol. However, results from laboratory tests and neuroimaging were sought from the participants' personal physicians when relevant to the diagnosis of cognitive impairment.

The ADAMS in-person evaluation was a 3- to 4-hour structured assessment conducted in the subject's residence by a nurse and NP technician. The following information about the respondent was collected from a knowledgeable informant and used as part of the diagnostic process: (1) a chronological history of cognitive symptoms; (2) medical history; (3) current medications; (4) current neuropsychiatric symptoms; (5) measures of severity of cognitive and functional impairment; and (6) family history of memory problems. The following information was collected from the subject and used as part of the diagnostic process: (1) a battery of NP measures (Table 4); (2) a self-report depression measure; (3) a standardized neurological examination; (4) a visual acuity measure; and (5) a 7-minute videotaped segment covering portions of the cognitive status and neurological examinations. Medical record releases were also sought to obtain relevant previous neuroimaging and laboratory results from the subjects' physicians.

A Duke University geropsychiatrist, neurologist, neuropsychologist, and cognitive neuroscientist reviewed all information collected during the in-home assessment, and assigned a preliminary research diagnosis regarding cognitive status. Final diagnoses were assigned by a consensus expert panel made up of neuropsychologists, neurologists, geropsychiatrists, and internists. The consensus panel reviewed each case and assigned a diagnosis in two stages, first without medical records and then with medical records.

Diagnoses fell within the three general categories of normal cognitive function, CIND, and dementia. Within the CIND and dementia categories, there were several subcategories to denote causes of cognitive impairment (Table 5). Diagnoses were assigned on the basis of clinical judgment, but they were anchored by established criteria. The DSMIII-R [55] and DSM-IV [13] criteria were used for dementia; the NINCDS-ADRDA criteria were used for AD [14], and other currently accepted diagnostic criteria were used for other subtypes of dementia. The DSM criteria and the NINCDS-ADRDA require evidence of impairment in memory and at least one other cognitive domain, and significant functional impairment in daily activities. Information on functional impairment was obtained from the informant, as self-report of this information may be of questionable validity.

The diagnostic criteria for CIND are still evolving, which has resulted in variability in the definition of mild impairment across studies. In an effort to use empirically based guidelines to define a CIND diagnosis, analyses were done before the start of ADAMS using data from other epidemiological studies of cognitive aging conducted by some of the same group of investigators $[65,68]$. On the basis of these analyses, operationalized criteria for CIND were developed. To capture the likely range of etiologies and clinical presentations of CIND, the condition was defined broadly as mild cognitive or functional impairment, reported by the participant or the informant, that did not meet criteria for dementia, or performance on NP measures that was below expectation and $\geq 1.5$ SD below published norms on any test. The diagnosis of CIND was anchored by this definition, but the final diagnosis was determined by clinical judgment. Across the three main diagnostic groups of normal cognition, CIND, and dementia, there was overlap in performance on the $16 \mathrm{NP}$ tests. Individuals with a diagnosis 
Table 4

ADAMS neuropsychological and other measures*

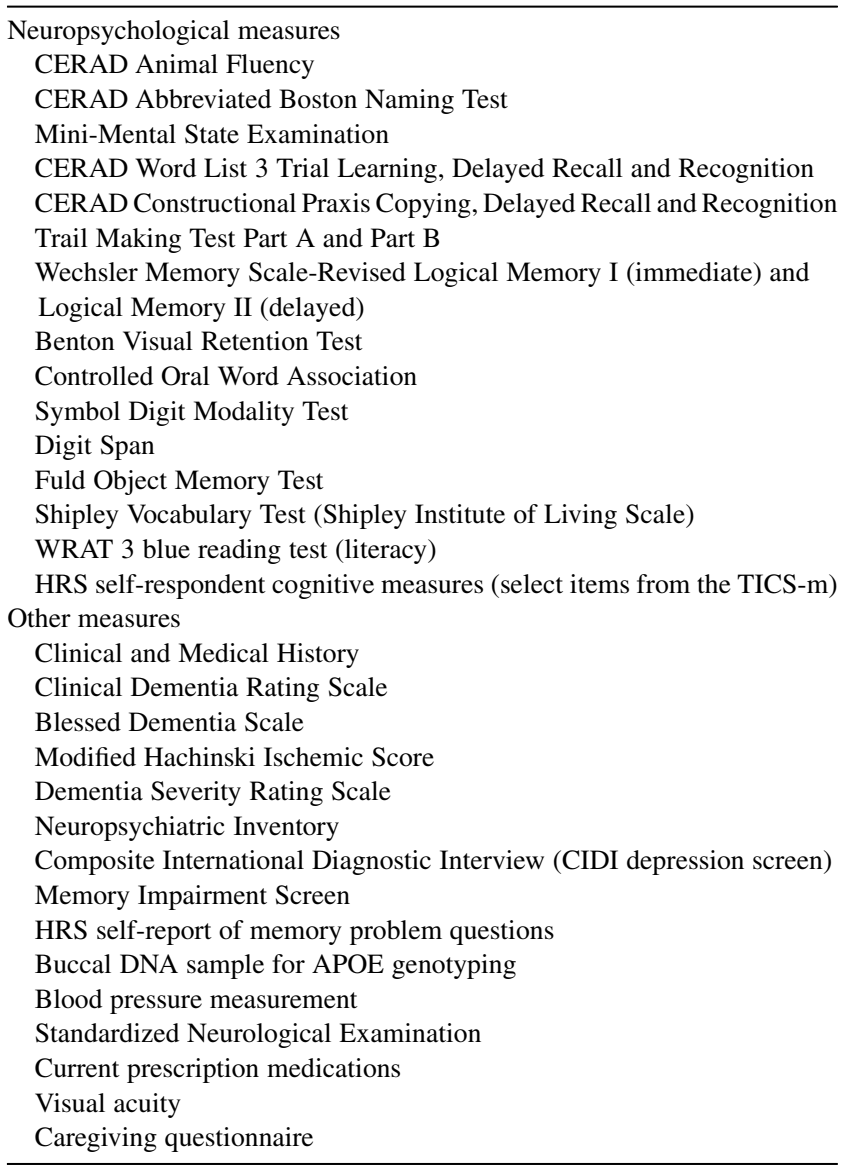

Abbreviations: ADAMS, Aging, Demographics, and Memory Study; APOE, Apolipoprotein E; CERAD, Consortium to Establish a Registry for Alzheimer's Disease; CIDI, Composite International Diagnostic Interview; HRS, Health and Retirement Study; TICS, Telephone Interview for Cognitive Status-modified; WRAT, Wide Range Achievement Test.

* Measures used at the initial ADAMS assessment; there were minor changes to the protocol used at the follow-up assessments.

of normal cognition scored at least in the mildly impaired range or worse ( $\geq 1.5 \mathrm{SD}$ below published norms) on an average of 2.2 tests (range of impaired tests: $0-12$ ). The individuals in the CIND diagnostic group performed in the mildly impaired or worse range on an average of 7.5 tests (range of impaired tests: 1-16 tests), whereas individuals with dementia scored in the impaired range on an average of 13.6 tests (range of impaired tests: 5-16). The overlap between the diagnostic groups in the number of tests with at least mildly impaired performance reflects the variation within each of these groups. It also highlights the limitation of normative values for interpretation of cognitive tests in a heterogeneous sample. These findings also point to the importance of using information on performance of daily activities, in addition to cognitive testing in the diagnostic process.

Several lines of evidence were explored to validate the operationalized diagnostic criteria used in the ADAMS. Us-
Table 5

ADAMS diagnostic categories

Demented
Alzheimer's disease
Probable AD
Possible AD
Vascular dementia
Probable vascular dementia
Possible vascular dementia
Subcortical dementias
Parkinson's disease
Huntington's disease
Progressive supranuclear palsy
Normal pressure hydrocephalus
Other dementias
Cognitive impairment, not demented
Prodromal Alzheimer's disease
Mild cognitive impairment
Cognitive impairment secondary to vascular disease
Stroke
Other neurological conditions
Other medical conditions
Depression
Psychiatric disorder
Low baseline intellect/mental retardation/learning disorder
Alcohol abuse (past)
Alcohol abuse (current)
Cognitive impairment, not demented, nonspecific
Normal cognitive function
Normal/noncase
Abrevations AD, Alteims

Abbreviations: AD, Alzheimer's disease; ADAMS, Aging, Demographics, and Memory Study.

ing data from other studies, the clinical diagnosis was compared with the "gold standard" neuropathological diagnosis for 175 subjects. The neuropathological confirmation of the clinical diagnosis of $\mathrm{AD}$ showed $93.0 \%$ sensitivity and $81.0 \%$ overall agreement [69]. These findings are comparable with those reported by university-based AD clinics $[48,70]$.

Data from follow-up assessments conducted in ADAMS provide validation of the CIND diagnoses. As part of the ADAMS protocol, a subset of 252 subjects completed a follow-up assessment approximately 18 months after the initial assessment. The same in-home assessment protocol was used for both the initial and follow-up assessments. Subjects were selected to receive a follow-up assessment for one of the following two reasons: (1) they received an initial diagnosis of CIND; or (2) they received an initial diagnosis of normal or demented, but the consensus panel considered the data collected at the initial assessment to be ambiguous and a second assessment providing longitudinal data would help to clarify the diagnosis. After the follow-up assessment, the consensus panel reviewed all of the information collected and then assigned a final diagnosis. At the time of the consensus review of the follow-up assessments, the panel of experts was blind to the diagnosis assigned after the first in-home assessment. However, the panel members were able to examine the clinical and NP data from the initial assessment as part of their deliberation regarding the diagnosis 
for the follow-up assessment. The most prevalent subtype of CIND was prodromal AD, defined as CIND with a pattern of clinical symptoms or performance on NP testing suggestive of prodromal $\mathrm{AD}$ and no other medical or neuropsychiatric conditions present to preclude an eventual diagnosis of AD. The second most prevalent subtype was CIND related to medical issues or sensory impairment.

Among individuals with a diagnosis of CIND at baseline and who completed the follow-up assessment, about $17.0 \%$ (weighted percent) progressed to dementia at the 18-month follow-up. In the CIND subcategory of prodromal AD, $28.0 \%$ (weighted) progressed to dementia in the same period, whereas only $9.0 \%$ (weighted) of those with CIND because of medical issues progressed to dementia. In contrast, only $9.0 \%$ of those with prodromal $\mathrm{AD}$ died before the follow-up assessment, but $21.0 \%$ of those with CIND due to medical issues died before follow-up. These differential rates of outcomes provide support for the ability of the ADAMS diagnostic methods to identify distinct CIND phenotypes with different prognoses. On average, about $20.0 \%$ (weighted) of those with CIND were categorized as cognitively normal at the 18-month follow-up, with the largest proportion of these being in the CIND group attributable to medical issues. Given the purported etiology for the cognitive problems in this latter group, it is not surprising that these individuals are the most likely to die or to return to normal cognitive status over time. Further validation of the operationalized diagnosis of CIND is provided by the stability of the diagnosis over time in most individuals. Overall, $64.0 \%$ of individuals who were reassessed after 18 months remained in the CIND group, whereas $54.0 \%$ of those in the prodromal $\mathrm{AD}$ group who were reassessed remained in the CIND diagnostic category. These results provide validation of the accuracy and stability of the ADAMS CIND diagnosis compared with diagnostic approaches for CIND used in other studies [71].

The ADAMS approach to operationalizing the diagnostic criteria for $\mathrm{AD}$ and CIND results in estimates of prevalence consistent with most other community-based studies. This provides some external validation for these procedures. Given this, one would expect that linkage of data from the ADAMS with detailed Health and Retirement Study longitudinal data on health, healthcare utilization, informal care, and economic resources and behavior should provide robust estimates of lifetime patient and caregiver costs of dementia in the population, as well as address other research questions on dementia and CIND that are best addressed in a population-representative sample.

Much of the information discussed in this section was originally published elsewhere [72].

\section{Discussion}

The conceptualization and diagnosis of $\mathrm{AD}$ are related, evolving processes, which are separated by a developmental lag. Current conceptualization of AD is rooted in autopsy findings of widespread neuritic plaques and neurofibrillary tangles first described in 1906 by Alois Alzheimer in a case with early symptom onset, subsequently generalized to late onset cases in 1968 when Blessed et al observed identical pathology in elderly persons [54]. For the past century, few clinical diagnosticians, much less epidemiologists, have had access to biomarkers (e.g., cerebrospinal fluid levels of abeta or phospho-tau, amyloid positron emission tomography imaging, or quantitative measures of medial temporal lobe atrophy). In the future, we can imagine novel approaches to the diagnosis of $\mathrm{AD}$, combining baseline burden and rate of change in behavioral, structural, molecular, and functional biomarkers [36-38]. However, currently available data on the prevalence and incidence of $\mathrm{AD}$ represent hard won investment in cohorts established using methods developed and implemented over at least the preceding 2 decades.

In determining prevalence and incidence in a target population, epidemiological studies must develop and implement methods to: (1) establish a representative study cohort, (2) ascertain cases for disease prevalence, (3) follow disease-free subjects to determine incident new cases, and (4) minimize and manage missing data at all levels. This article focuses on methods used for the operational ascertainment of cases, namely for $\mathrm{AD}$, dementia, and other agerelated cognitive impairment. Many considerations related to case ascertainment are summarized in Table 3.

The process of ascertaining AD in epidemiological studies typically represents a two-step process as follows: (1) Does the case meet a threshold of severity in cognitive or functional impairment, and (2) What is the etiological cause of the cognitive impairment? Step 1 is usually standardized using NP testing. Although the choice of specific tests may vary, there is general consensus on the most important cognitive domains and the need for appropriate norms. The choices of specific tests were not identified as major sources of variance between studies. Table 4 shows examples of NP tests used in ADAMS (see also Wilson et al [73]). In contrast, considerable challenges (not addressed here) could be anticipated in identifying appropriate NP tests for different cultural, ethnic, socioeconomic, and education groups [64]. Step 2 has depended for the most part on the operationalization of the following:(1) NINCDS-ADRDA criteria for probable or possible AD [14], (2) DSM-III [74], DSM-III-R [55], or DSM-IV [13] criteria for dementia of the Alzheimer type, and (3) recently on definitions of MCI-amnestic type $[19,20]$. Limited autopsy data are available from some epidemiological studies to assess the sensitivity and specificity of etiological diagnoses.

The selection of different cut-offs for severity of cognitive impairment (step 1) was identified as the single most important factor affecting estimates of prevalence and incidence of AD. As the cut-point is moved toward less severe impairment, the prevalence of $\mathrm{AD}$ will increasewith potentially fewer false negatives (higher sensitivity), but potentially more false positives (lower specificity). 
Prevalence refers to the total number of cases in the population at a given time, and reflects the combined effects of incidence and duration of disease. Studies using more sensitive definitions of cognitive impairment (e.g., EBSHP/ CHAP's reliance on cognitive testing, without reference to functional decline) will lead to higher prevalence estimates of dementia than those using less sensitive cut-offs (e.g., FHS initially focused on moderate to severe dementia). After adding mild dementia cases in FHS, the prevalence of all cause dementia almost doubled from $1.1 \%$ to $1.9 \%$ for persons 75 years old, and increased from $44.4 \%$ to $57.4 \%$ at age 100 years (Fig. 1). The effects on prevalence resulting from shifting the threshold between MCI and dementia is illustrated in data from the MCSA (Fig. 3) and could be generalized to shifting boundaries between $\mathrm{MCI}$ and normal aging. The shift to earlier diagnosis of AD during stages of MCI (even early MCI) has similar implications. As shown in MCSA [52] and FHS, inclusion of MCI will significantly increase the prevalence of $\mathrm{AD}$, depending on cut-offs. These data clearly illustrate major effects on prevalence because of differences in the threshold of cognitive impairment selected for case ascertainment.

Incidence refers to the number of newly diagnosed cases during a specified period. Because persons with AD can be assumed to follow a progressive trajectory, earlier diagnosis of $\mathrm{AD}$ (e.g., including amnestic MCI or pre-MCI) should shift age-specific incidence rates to earlier ages. In the FHS, moving the threshold from moderate to mild dementia increased the 25-year cumulative incidence of dementia (adjusted for competing mortality) from $12.2 \%$ to $20.9 \%$ (Table 2). Subjects in the BLSA were highly educated volunteers, which could have delayed detection of dementia and shifted incidence rates to the right. However, the slope of agerelated incident dementia in BLSA is quite similar to those reported from Framingham, East Boston, and Rochester (Fig. 6). No differences in dementia incidence are reported for women and men in the FHS or the 90+ Study. However, women live longer in general and live longer with dementia, explaining their higher cumulative-incident lifetime risk and prevalence estimates $[59,60]$.

Step 2 (diagnosis of etiological subtype, namely AD) is usually based on the application of NINCDS-ADRDA [14] or DSM criteria $[13,55,74]$ with data from medical history and clinical examination, often without biomarkers or imaging studies, and rarely validated against autopsy diagnoses. Less than satisfactory is the criterion that a diagnosis of probable AD per NINDS-ADRDA criteria depends on the exclusion of any other disorder that is likely to cause the dementia syndrome. Thus, the diagnosis of AD depends on vigilance, diligence, and cost of excluding other possible causes. It is not difficult to exclude common systemic metabolic disorders or symptomatic stroke, but quite challenging to diagnose other neurodegenerative conditions (e.g., dementia with Lewy bodies, subclinical vascular disease, or mixed pathologies). The NINCDS-ADRDA criteria for possible $\mathrm{AD}$ allow for atypical presentations in course or mixed etiologies. Evans and Wilson (section 3) suggest that forced choice between $\mathrm{AD}$ versus non- $\mathrm{AD}$ (without intermediate category of possible or mixed), as is often typical in epidemiological surveys, may lend bias toward diagnosis of AD. No consideration is given in this article to the comparisons of estimates for probable versus possible AD. Clearly, however, the inclusion of possible AD would lead to large increases in both prevalence and incidence rates.

In a few studies (not reviewed here), excellent autopsy rates have been obtained which are as follows: HonoluluAsia Aging Study (21\%-34\%) [75], Religious Orders Study (ROS; 94\%) [47], and Rush Memory and Aging Project (MAP; 85\%) [47]. In Alzheimer Disease Centers memory clinics, the NINCDS-ADRDA and DSM-III criteria for dementia versus a neuropathological gold standard was found to be $93 \%$ sensitive, but only $55 \%$ specific [76]. In one neuropathological study of 175 cases drawn from three epidemiological samples, a lenient clinical diagnosis of $\mathrm{AD}$ was associated with $93.0 \%$ sensitivity, but only $19 \%$ specificity [69]. In the combined autopsy series from the ROS and MAP, of 179 persons with probable $\mathrm{AD}, 90 \%$ had pathologically confirmed $\mathrm{AD}$, but nearly one-half (46\%) had mixed pathologies, which also contributed significantly to risk of dementia [47]. Comparison of neuropathological diagnoses showed less severe AD pathology, more cerebral infarcts, and more mixed pathologies in these two community-based versus clinical cohorts [77]. In the 90+ Study, half of demented autopsy cases proved to have minimal AD pathology [61]. Thus, recent data from community-based clinical-pathological studies indicate a tendency to underestimate the presence of mixed pathologies in cases clinically diagnosed with probable $\mathrm{AD}$.

The relationship between MCI and etiological subtype (step 2) is only briefly touched in this review. Association between MCI/CIND subtype and rate of progression or conversion to dementia, as described in section 6, may provide some form of face validity. However, autopsies from epidemiological cohorts are needed to validate the correspondence between MCI subtypes and neuropathological diagnoses. Schneider et al observed more infarcts in community-based versus clinicbased samples of MCI [77]. Of 134 persons with MCI, approximately half (54\%) had pathologically diagnosed AD (58.7\% amnestic; $49.2 \%$ nonamnestic); $19 \%$ had mixed pathologies (22.7\% amnestic; $15.3 \%$ nonamnestic) [47]. Thus, MCI is an even more pathologically heterogeneous process, with mixed pathologies contributing additively to cognitive impairment. As the focus shifts to earlier detection of AD as MCI and pre-MCI, specific etiological biomarkers are needed.

In summary, this article illustrates considerable awareness and understanding about the effects exerted by various operational diagnoses on estimates of prevalence and incidence rates of MCI/CIND or dementia in, mainly, middle-class white Americans. The single most important factor affecting estimates of $\mathrm{AD}$ prevalence is the severity of cognitive impairment used for case ascertainment. Fortunately, differences in severity level have less effect on estimates of 
incidence. Recent neuropathological findings from the ROS and MAP indicate that a clinical diagnosis of probable AD is usually associated with $\mathrm{AD}$ pathology, either alone or in combination with other pathologies, whereas a diagnosis of $\mathrm{MCI}$ is even more heterogeneous. Incremental progress in accuracy of etiological diagnosis can be anticipated with the addition of molecular and structural biomarkers in the next generation of epidemiological studies.

\section{Acknowledgments}

The FHS was supported by a National Heart, Lung, and Blood Institute contract (N01HC25195), by grants from the National Institute of Neurological Disorders and Stroke (R01NS17950), and from the National Institute on Aging (R01AG16495, R01AG08122, R01AG033193, R01AG031287, R01AG033040). The EBSHP was supported by National Institute on Aging contracts (N01AG12106, N01AG02107) and CHAP was supported by a grant from the same institute (R01AG011101). The MCSA was supported by National Institute on Aging grants (U01AG06786, P50AG016574, and R01AG034676). The project was also supported by Robert H. and Clarice Smith, and Abigail Van Buren Alzheimer's Disease Research Program at the Mayo Clinic, Rochester, Minnesota. Knopman, Petersen, and Rocca thank their colleagues on this project for their contributions: Rosebud O. Roberts, Yonas E. Geda, V. Shane Pankratz, Bradley F. Boeve, Robert J. Ivnik, and Eric G. Tangalos. The BLSA and the $90+$ Study were supported by National Institute on Aging (R01AG008325, R01AG021055). The BLSA is a research project of the Intramural Division of the NIA. Kawas and Corrada thank their colleagues, the research participants, and families of each study. The ADAMS is a component of the Health and Retirement Study, which is supported by the National Institute on Aging (U01 AG009740). Chui is supported by the National Institute on Aging (P01AG012435, P50AG005142).

\section{References}

[1] Brookmeyer R, Evans DA, Hebert LA, Langa KM, Heeringa SG, Plassman BL, et al. National estimates of the prevalence of Alzheimer's disease in the United States. Alzheimers Dement 2011; 7:61-73.

[2] Dawber TR, Kannel WB. The Framingham study. An epidemiological approach to coronary heart disease. Circulation 1966;34:553-5.

[3] Farmer ME, White LR, Kittner SJ, Kaplan E, Moes E, McNamara P, et al. Neuropsychological test performance in Framingham: a descriptive study. Psychol Rep 1987;60:1023-40.

[4] Folstein MF, Folstein SE, McHugh PR. "Mini-mental state." A practical method for grading the cognitive state of patients for the clinician. J Psychiatr Res 1975;12:189-98.

[5] Feinleib M, Kannel WB, Garrison RJ, McNamara PM, Castelli WP. The Framingham Offspring Study. Design and preliminary data. Prev Med 1975;4:518-25.

[6] Bachman DL, Wolf PA, Linn RT, Knoefel JE, Cobb JL, Belanger AJ, et al. Incidence of dementia and probable Alzheimer's disease in a general population: the Framingham Study. Neurology 1993;43:515-9.
[7] Bachman DL, Wolf PA, Linn R, Knoefel JE, Cobb J, Belanger A, et al. Prevalence of dementia and probable senile dementia of the Alzheimer type in the Framingham Study. Neurology 1992;42:115-9.

[8] Beiser A, D'Agostino RB Sr, Seshadri S, Sullivan LM, Wolf PA. Computing estimates of incidence, including lifetime risk: Alzheimer's disease in the Framingham Study. The Practical Incidence Estimators (PIE) macro. Stat Med 2000;19:1495-522.

[9] Seshadri S, Wolf PA, Beiser A, Au R, McNulty K, White R, et al. Lifetime risk of dementia and Alzheimer's disease. The impact of mortality on risk estimates in the Framingham Study. Neurology 1997; 49:1498-504.

[10] Seshadri S, Beiser A, Kelly-Hayes M, Kase CS, Au R, Kannel WB, et al. The lifetime risk of stroke: estimates from the Framingham Study. Stroke 2006;37:345-50.

[11] Seshadri S, Wolf PA. Lifetime risk of stroke and dementia: current concepts, and estimates from the Framingham Study. Lancet Neurol 2007;6:1106-14.

[12] Berg L. Clinical dementia rating (CDR). Psychopharmacol Bull 1988; 24:637-9.

[13] American Psychiatric Association. Diagnostic and Statistical Manual of Mental Disorders: DSM-IV. 4th ed. Washington, DC: American Psychiatric Association; 1994.

[14] McKhann G, Drachman D, Folstein M, Katzman R, Price D, Stadlan EM. Clinical diagnosis of Alzheimer's disease: report of the NINCDS-ADRDA Work Group under the auspices of Department of Health and Human Services Task Force on Alzheimer's Disease. Neurology 1984;34:939-44.

[15] Chui HC, Victoroff JI, Margolin D, Jagust W, Shankle R, Katzman R, et al. Criteria for the diagnosis of ischemic vascular dementia proposed by the State of California Alzheimer's Disease Diagnostic and Treatment Centers. Neurology 1992;42:473-80.

[16] Roman GC, Tatemichi TK, Erkinjuntti T, Cummings JL, Masdeu JC, Garcia JH, et al. Vascular dementia: diagnostic criteria for research studies. Report of the NINDS-AIREN International Workshop. Neurology 1993;43:250-60.

[17] McKeith IG, Perry EK, Perry RH. Report of the second dementia with Lewy body international workshop: diagnosis and treatment. Consortium on Dementia with Lewy Bodies. Neurology 1999;53:902-5.

[18] Miller BL, Ikonte C, Ponton M, Levy M, Boone K, Darby A, et al. A study of the Lund-Manchester research criteria for frontotemporal dementia: clinical and single-photon emission CT correlations. Neurology 1997;48:937-42.

[19] Petersen RC, Smith GE, Waring SC, Ivnik RJ, Tangalos EG, Kokmen E. Mild cognitive impairment: clinical characterization and outcome. Arch Neurol 1999;56:303-8.

[20] Winblad B, Palmer K, Kivipelto M, Jelic V, Fratiglioni L, Wahlund LO, et al. Mild cognitive impairment—-beyond controversies, towards a consensus: report of the International Working Group on Mild Cognitive Impairment. J Intern Med 2004;256:240-6.

[21] Elias MF, Beiser A, Wolf PA, Au R, White RF, D'Agostino RB. The preclinical phase of Alzheimer disease: a 22-year prospective study of the Framingham Cohort. Arch Neurol 2000;57:808-13.

[22] Evans DA, Funkenstein HH, Albert MS, Scherr PA, Cook NR, Chown MJ, et al. Prevalence of Alzheimer's disease in a community population of older persons. Higher than previously reported. JAMA 1989;262:2551-6.

[23] Evans DA, Bennett DA, Wilson RS, Bienias JL, Morris MC, Scherr PA, et al. Incidence of Alzheimer disease in a biracial urban community: relation to apolipoprotein E allele status. Arch Neurol 2003;60:185-9.

[24] Wilson RS, Beckett LA, Bennett DA, Albert MS, Evans DA. Change in cognitive function in older persons from a community population: relation to age and Alzheimer disease. Arch Neurol 1999;56:1274-9.

[25] Wilson RS, Aggarwal NT, Barnes LL, Mendes de Leon CF, Hebert LE, Evans DA. Cognitive decline in incident Alzheimer disease in a community population. Neurology 2010;74:951-5. 
[26] Gijsen R, Hoeymans N, Schellevis FG, Ruwaard D, Satariano WA, van den Bos GA. Causes and consequences of comorbidity: a review. J Clin Epidemiol 2001;54:661-74.

[27] Fillenbaum GG, Pieper CF, Cohen HJ, Cornoni-Huntley JC, Guralnik JM. Comorbidity of five chronic health conditions in elderly community residents: determinants and impact on mortality. J Gerontol A Biol Sci Med Sci 2000;55:M84-9.

[28] Albert M, Smith LA, Scherr PA, Taylor JO, Evans DA, Funkenstein HH. Use of brief cognitive tests to identify individuals in the community with clinically diagnosed Alzheimer's disease. Int J Neurosci 1991;57:167-78.

[29] Meinert CL, Tonascia S. Clinical trials: design, conduct, and analysis. New York, NY: Oxford University Press; 1986.

[30] Kawas C, Gray S, Brookmeyer R, Fozard J, Zonderman A. Age-specific incidence rates of Alzheimer's disease: the Baltimore Longitudinal Study of Aging. Neurology 2000;54:2072-7.

[31] Petersen RC, Roberts RO, Knopman DS, Boeve BF, Geda YE, Ivnik RJ, et al. Mild cognitive impairment: ten years later. Arch Neurol 2009;66:1447-55.

[32] Jicha GA, Parisi JE, Dickson DW, Johnson K, Cha R, Ivnik RJ, et al. Neuropathologic outcome of mild cognitive impairment following progression to clinical dementia. Arch Neurol 2006; 63:674-81.

[33] Shaw LM, Vanderstichele H, Knapik-Czajka M, Clark CM, Aisen PS, Petersen RC, et al. Cerebrospinal fluid biomarker signature in Alzheimer's disease neuroimaging initiative subjects. Ann Neurol 2009; 65:403-13.

[34] Jagust WJ, Landau SM, Shaw LM, Trojanowski JQ, Koeppe RA, Reiman EM, et al. Relationships between biomarkers in aging and dementia. Neurology 2009;73:1193-9.

[35] Petersen RC, Aisen PS, Beckett LA, Donohue MC, Gamst AC, Harvey DJ, et al. Alzheimer's Disease Neuroimaging Initiative (ADNI): clinical characterization. Neurology 2010;74:201-9.

[36] Petersen RC. Alzheimer's disease: progress in prediction. The Lancet Neurology 2010;9:4-5.

[37] Jack CR Jr, Knopman DS, Jagust WJ, Shaw LM, Aisen PS, Weiner MW, et al. Hypothetical model of dynamic biomarkers of the Alzheimer's pathological cascade. Lancet Neurol 2010;9:119-28.

[38] Visser PJ, Verhey F, Knol DL, Scheltens P, Wahlund LO, FreundLevi Y, et al. Prevalence and prognostic value of CSF markers of Alzheimer's disease pathology in patients with subjective cognitive impairment or mild cognitive impairment in the DESCRIPA study: a prospective cohort study. Lancet Neurol 2009;8:619-27.

[39] Roberts RO, Geda YE, Knopman DS, Cha RH, Pankratz VS, Boeve BF, et al. The Mayo Clinic Study of Aging: design and sampling, participation, baseline measures and sample characteristics. Neuroepidemiology 2008;30:58-69.

[40] Kokmen E, Beard CM, Offord KP, Kurland LT. Prevalence of medically diagnosed dementia in a defined United States population: Rochester, Minnesota, January 1, 1975. Neurology 1989;39:773-6.

[41] Khachaturian ZS. Diagnosis of Alzheimer's disease: two-decades of progress. J Alzheimers Dis 2006;9:409-15.

[42] Mirra SS, Heyman A, McKeel D, Sumi SM, Crain BJ, Brownlee LM, et al. The Consortium to Establish a Registry for Alzheimer's Disease (CERAD). Part II. Standardization of the neuropathologic assessment of Alzheimer's disease. Neurology 1991;41:479-86.

[43] Brookmeyer R, Gray S, Kawas C. Projections of Alzheimer's disease in the United States and the public health impact of delaying disease onset. Am J Public Health 1998;88:1337-42.

[44] Knopman DS, Parisi JE, Salviati A, Floriach-Robert M, Boeve BF, Ivnik RJ, et al. Neuropathology of cognitively normal elderly. J Neuropathol Exp Neurol 2003;62:1087-95.

[45] Davis DG, Schmitt FA, Wekstein DR, Markesbery WR. Alzheimer neuropathologic alterations in aged cognitively normal subjects. J Neuropathol Exp Neurol 1999;58:376-88.

[46] Gearing M, Mirra SS, Hedreen JC, Sumi SM, Hansen LA, Heyman A. The Consortium to Establish a Registry for Alzheimer's Disease (CE-
RAD). Part X. Neuropathology confirmation of the clinical diagnosis of Alzheimer's disease. Neurology 1995;45:461-6.

[47] Schneider JA, Arvanitakis Z, Leurgans SE, Bennett DA. The neuropathology of probable Alzheimer disease and mild cognitive impairment. Ann Neurol 2009;66:200-8.

[48] Lim A, Tsuang D, Kukull W, Nochlin D, Leverenz J, McCormick W, et al. Clinico-neuropathological correlation of Alzheimer's disease in a community-based case series. J Am Geriatr Soc 1999;47:564-9.

[49] Ikonomovic MD, Klunk WE, Abrahamson EE, Mathis CA, Price JC, Tsopelas ND, et al. Post-mortem correlates of in vivo PiB-PET amyloid imaging in a typical case of Alzheimer's disease. Brain 2008; 131:1630-45.

[50] Klunk WE, Engler H, Nordberg A, Wang Y, Blomqvist G, Holt DP, et al. Imaging brain amyloid in Alzheimer's disease with Pittsburgh Compound-B. Ann Neurol 2004;55:306-19.

[51] Okello A, Koivunen J, Edison P, Archer HA, Turkheimer FE, Nagren K, et al. Conversion of amyloid positive and negative $\mathrm{MCI}$ to $\mathrm{AD}$ over 3 years: an 11C-PIB PET study. Neurology 2009;73:754-60.

[52] Petersen RC, Roberts RO, Knopman DS, Geda YE, Cha RH, Pankratz VS, et al. Prevalence of mild cognitive impairment is higher in men: the Mayo Clinic Study of Aging. Neurology 2010;75:889-97.

[53] Lorenzi M, Donohue M, Paternico D, Scarpazza C, Ostrowitzki S, Blin O, et al. Enrichment through biomarkers in clinical trials of Alzheimer's drugs in patients with mild cognitive impairment. Neurobiol Aging 2010;31:1443-51, $1451 \mathrm{e} 1$.

[54] Blessed G, Tomlinson BE, Roth M. The association between quantitative measures of dementia and of senile change in the cerebral grey matter of elderly subjects. Br J Psychiatry 1968;114:797-811.

[55] American Psychiatric Association, American Psychiatric Association Work Group to Revise DSM-III. Diagnostic and Statistical Manual of Mental Disorders: DSM-III-R. 3rd ed. Washington, DC: American Psychiatric Association; 1987.

[56] Hebert LE, Scherr PA, Beckett LA, Albert MS, Pilgrim DM, Chown MJ, et al. Age-specific incidence of Alzheimer's disease in a community population. JAMA 1995;273:1354-9.

[57] Katzman R, Aronson M, Fuld P, Kawas C, Brown T, Morgenstern H, et al. Development of dementing illnesses in an 80-year-old volunteer cohort. Ann Neurol 1989;25:317-24.

[58] Eisdorfer C, Cohen D. Diagnostic criteria for primary neuronal degeneration of the Alzheimer's type. J Fam Pract 1980;11:553-7.

[59] Corrada MM, Brookmeyer R, Berlau D, Paganini-Hill A, Kawas CH. Prevalence of dementia after age 90: results from the 90+ study. Neurology 2008;71:337-43.

[60] Corrada MM, Brookmeyer R, Paganini-Hill A, Berlau D, Kawas CH. Dementia incidence continues to increase with age in the oldest old: the 90+ study. Ann Neurol 2010;67:114-21.

[61] Berlau DJ, Corrada MM, Head E, Kawas CH. APOE $\varepsilon 2$ is associated with intact cognition but increased Alzheimer pathology in the oldest old. Neurology 2009;72:829-34.

[62] Crystal HA, Dickson D, Davies P, Masur D, Grober E, Lipton RB. The relative frequency of "dementia of unknown etiology" increases with age and is nearly 50\% in nonagenarians. Arch Neurol 2000;57:713-9.

[63] Savva GM, Wharton SB, Ince PG, Forster G, Matthews FE, Brayne C, et al. Age, neuropathology, and dementia. N Engl J Med 2009; 360:2302-9.

[64] Mayeux R, Reitz C, Brickman AM, Haan MN, Manly JJ, Glymour MM, et al. Operationalizing diagnostic criteria for Alzheimer's disease and other age-related cognitive impairment-Part 1. Alzheimers Dement 2011;7:15-34.

[65] Plassman BL, Havlik RJ, Steffens DC, Helms MJ, Newman TN, Drosdick D, et al. Documented head injury in early adulthood and risk of Alzheimer's disease and other dementias. Neurology 2000;55:1158-66.

[66] Breitner JC, Welsh KA, Gau BA, McDonald WM, Steffens DC, Saunders AM, et al. Alzheimer's disease in the National Academy of Sciences-National Research Council Registry of Aging Twin Veterans. III. Detection of cases, longitudinal results, and observations on twin concordance. Arch Neurol 1995;52:763-71. 
[67] Breitner JC, Wyse BW, Anthony JC, Welsh-Bohmer KA, Steffens DC, Norton MC, et al. APOE-epsilon4 count predicts age when prevalence of AD increases, then declines: the Cache County Study. Neurology 1999;53:321-31.

[68] Plassman BL, Steffens DC, Burke JR, Welsh-Bohmer KA, Newman TN, Drosdick D, et al. Duke Twins Study of Memory in Aging in the NAS-NRC Twin Registry. Twin Res Hum Genet 2006;9:950-7.

[69] Plassman BL, Khachaturian AS, Townsend JJ, Ball MJ, Steffens DC, Leslie CE, et al. Comparison of clinical and neuropathologic diagnoses of Alzheimer's disease in 3 epidemiologic samples. Alzheimers Dement 2006;2:2-11.

[70] Massoud F, Devi G, Stern Y, Lawton A, Goldman JE, Liu Y, et al. A clinicopathological comparison of community-based and clinicbased cohorts of patients with dementia. Arch Neurol 1999; 56:1368-73.

[71] Matthews FE, Stephan BC, McKeith IG, Bond J, Brayne C. Medical Research Council Cognitive Function and Ageing Study. Two-year progression from mild cognitive impairment to dementia: to what extent do different definitions agree? J Am Geriatr Soc 2008; 56:1424-33.
[72] Langa KM, Plassman BL, Wallace RB, Herzog AR, Heeringa SG, Ofstedal MB, et al. The Aging, Demographics, and Memory Study: study design and methods. Neuroepidemiology 2005;25:181-91.

[73] Wilson RS, Weir DR, Leurgans S, Evans DA, Hebert LE, Langa KM, et al. Sources of variability in estimates of the prevalence of Alzheimer's disease in the United States. Alzheimer Dement (in press).

[74] American Psychiatric Association. Diagnostic and Statistical Manual of Mental Disorders (DSM III). Washington, DC: American Psychiatric Association; 1980

[75] Petrovitch H, Ross GW, He Q, Uyehara-Lock J, Markesbery W, Davis D, et al. Characterization of Japanese-American men with a single neocortical AD lesion type. Neurobiol Aging 2008;29:1448-55.

[76] Mayeux R, Saunders AM, Shea S, Mirra S, Evans D, Roses AD, et al. Utility of the apolipoprotein $\mathrm{E}$ genotype in the diagnosis of Alzheimer's disease. Alzheimer's Disease Centers Consortium on Apolipoprotein E and Alzheimer's Disease. N Engl J Med 1998; 338:506-11. Erratum in: N Engl J Med 1998;338:1325.

[77] Schneider JA, Aggarwal NT, Barnes L, Boyle P, Bennett DA. The neuropathology of older persons with and without dementia from community versus clinic cohorts. J Alzheimers Dis 2009;18:691-701. 